\title{
Reuse of oak chips for modification of the volatile fraction of alcoholic beverages
}

\author{
Eduardo Coelho ", José A. Teixeira, Teresa Tavares, Lucília Domingues, José M. Oliveira \\ CEB - Centre of Biological Engineering, University of Minho, 4710-057, Braga, Portugal
}

\section{A R T I C L E I N F O}

\section{Keywords:}

Wood ageing

Barrel alternatives

Volatile compounds

Sensory properties

\begin{abstract}
A B S T R A C T
New or used barrels can be applied in ageing of alcoholic beverages. Compounds adsorbed in wood migrate between beverages along with wood extractives. As barrel ageing is costly and time-consuming, processes using wood fragments have been gaining interest. These generate wood residues for which the reuse is still not well established. This work aims at the reuse of oak fragments for the additive ageing of alcoholic beverages. Oak chips, previously immersed in fortified wine, were applied to beer, wine and grape marc spirit. Wood compounds and adsorbed wine volatiles were extracted, with more impact and satisfactory yields on beer composition. Also, wood adsorbed beverages compounds in a subtractive ageing phenomena. Beer formulations using different binomial wood concentration/temperature combinations were generated and presented to trained tasters. Higher temperatures and wood concentrations led to prominence of wood descriptors and lower perception of fruity and floral aromas, reflecting the changes in chemical composition.
\end{abstract}

\section{Introduction}

Wood ageing is commonly used as a strategy to modify and enhance the composition of alcoholic beverages. Quite a few alcoholic beverages traditionally resort to ageing in contact with wood, either beer, wine or spirits. During contact with wood, several ageing phenomena can occur namely additive ageing - the extraction of wood constituents to the beverage - (Canas, Caldeira, \& Belchior, 2013; García-Estévez, Alcalde-Eon, Le Grottaglie, Rivas-Gonzalo, \& Escribano-Bailón, 2015), subtractive ageing - the loss of compounds due to evaporation or wood sorption - (Barrera-García et al., 2007; Coelho, Domingues, Teixeira, Oliveira, \& Tavares, 2019), chemical ageing - reaction between beverage and wood components - (Coetzee \& Du Toit, 2015) and biological ageing - conversion of constituents in the matrix due to the action of microbes - (Coelho et al., 2020; Snauwaert et al., 2016). Traditionally, ageing is performed by storing the beverage in wood casks or barrels during periods of time dependent on the beverage to be aged and the intended product. Oak is the most preferred wood for barrel production and beverage ageing due to its mechanical properties and its capacity to act as a dynamic container (Álamo-Sanza \& Nevares, 2018). Also it imparts beverage composition with several compounds that positively contribute to the sensory characteristics of aged beverages such as furan compounds, volatile phenols, tannins, lactones among several other (Mosedale \& Puech, 2003). During its lifecycle, cooperage wood can be used, reused, regenerated and eventually discarded. For barrels, there is a well-established reuse flow depending on the aged beverages. Wine production usually resorts to new barrels, which may be reused depending on their extractive content (Zamora, 2019). Bourbon production also resorts to new barrels, whereas Scotch and Irish Whiskey production usually resort to previously used ones, either from Bourbon and Brandy production, or from fortified wine production, mainly Sherry but also Port and Madeira wines (Quinn, 2014). Beer ageing also resorts to reused casks, either from Bourbon or Sherry wine production (Spitaels et al., 2014). Previous works have demonstrated that oak wood adsorbs compounds from the beverages during ageing (Coelho, Teixeira, Domingues, Tavares, \& Oliveira, 2019), which are transported to other matrices in subsequent contacts (Coelho, Teixeira, et al., 2019). Transference of characteristic wine compounds such as quercetin-glucuronide and myricetin-glucoside, has also been identified in whiskies aged in Sherry casks, which were differentiated in their chemical composition from whiskies aged in Bourbon casks (Roullier-Gall et al., 2018).

As an alternative to barrel ageing, several processes have been developed envisaging intensification of ageing phenomena, by immersing oak fragments in the beverage in combination with other physical-chemical treatments. Several efforts have been made for

\footnotetext{
* Corresponding author.

E-mail address: e.coelho@ceb.uminho.pt (E. Coelho).
} 
acceleration of wine ageing (Tao, García, \& Sun, 2014), as well as ageing of several spirits, namely grape marc distillate (Rodriguez-Solana, Rodriguez-Freigedo, Salgado, Domínguez, \& Cortés-Diéguez, 2017), brandy (Canas, Caldeira, Anjos, \& Belchior, 2019; Schwarz, Rodríguez, Sánchez, Guillén, \& Barroso, 2014) and whisky (Pielech-Przybylska \& Balcerek, 2019). A few works have also envisaged extraction of oak compounds to beer, by applying oak chips (Sterckx, Saison, \& Delvaux, 2012a; 2012b; Wyler, Angeloni, Alcarde, \& da Cruz, 2015) and further on combining wood with microorganisms for biological ageing (Coelho et al., 2020). Accelerated ageing technologies often make use of oak alternatives such as chips, staves, cubes or sticks (Chatonnet, 2007), which are a cheaper, eco-friendlier and a more sustainable alternative for ageing (García-Alcaraz et al., 2020). With the dissemination of these technologies, the use and demand of barrel alternative cooperage products is expected to increase, as well as the generation of their used residues, for which a reuse circuit is still not well established.

Moreover, research on beverage ageing mainly focuses the transference of oak compounds to the beverage along with the occurring chemical and biological transformations, whereas the contribution of wood reuse is often overlooked. This work aims to investigate the contribution of used wood to the volatile fraction of beverages, when reused for induction of aroma compounds in accelerated ageing processes. The main issue under study is the transference of compounds from one beverage to another through wood reutilization, which modifies not only the chemical composition but also the sensory properties of the beverage. Thus, revalorization of used wood fragments is proposed and demonstrated in order to promote their reuse in accelerated ageing processes.

\section{Materials and methods}

\subsection{Materials and chemicals}

The following compounds with the corresponding purities were used as standards for the GC-MS analyses. From Fluka: isobutyl acetate (99\%), ethyl pentanoate ( $\geq 99.7 \%$ ), ethyl phenylacetate ( $\geq 99 \%$ ), 2-phenylethyl acetate ( $\geq 99 \%$ ), ethyl dodecanoate ( $\geq 98 \%$ ), 2-methyl-1-propanol ( $\geq 99.9 \%)$, 2-methyl-1-butanol ( $\geq 98 \%)$, 3-methyl-1-butanol ( $\geq 99.8 \%)$, 1-hexanol ( $\geq 99.9 \%), \quad Z$-3-hexenol ( $\geq 98 \%)$, 1-octanol ( $\geq 99.5 \%)$, 2-phenylethanol ( $\geq 99 \%$ ), propanoic acid $(\geq 99.5 \%)$, butanoic acid ( $\geq 99.5 \%)$, hexanoic acid $(\geq 98 \%)$, decanoic acid $(\geq 98 \%)$, dodecanoic acid ( $\geq 99 \%)$, furfural (99\%), linalool furanic oxide $(\geq 97 \%)$, benzaldehyde ( $\geq 99 \%$ ), vanillin $(\geq 98 \%)$ and acetoin $(97 \%)$; from Aldrich: ethyl butyrate (99\%), ethyl-2-methylbutyrate (99\%), ethyl-3methylbutyrate $(98 \%)$, isoamyl acetate $(\geq 99 \%)$, ethyl hexanoate ( $\geq 99 \%)$, hexyl acetate $(99 \%)$, ethyl heptanoate (99\%), ethyl lactate (99\%), ethyl octanoate ( $\geq 99 \%$ ), ethyl-3-hydroxybutyrate (99\%), ethyl decanoate $(\geq 99 \%)$, diethyl succinate $(99 \%)$, methyl salycilate $(\geq 99 \%)$, diethyl malate $(\geq 97 \%)$, ethyl cinnamate (99\%), E-3-hexenol (96\%), benzyl alcohol ( $\geq 99.9 \%$ ), linalool (97\%), $\beta$-citronellol (95\%), isovaleric acid (99\%), octanoic acid ( $\geq 99.5 \%)$, 5-methylfurfural (99\%), cis/transoak lactone $(\geq 98 \%), \quad \gamma$-nonalactone $(\geq 98 \%), \quad \gamma$-ethoxycarbonyl$\gamma$-butyrolactone (95\%), syringaldehyde (98\%), octanal (99\%), nonanal (95\%), 4-methylguaiacol ( $\geq 98 \%$ ), eugenol (99\%), 4-ethylphenol (99\%), 4-vinylguaiacol (98\%), 2,6-dimethoxyphenol (99\%), 4-vinylphenol (10\%), acetovanillone (98\%), tyrosol (98\%), methionol (98\%) and $\beta$-damascenone (1.3\%); $\alpha$-terpineol and acetic acid $(\geq 99.8 \%)$ from Merck, cis-nerolidol (98\%) and 4-ethylguaiacol (98\%) from Alfa Aesar and 5-hydroxymethylfurfural from Acros Organics.

For generating the used oak wood, American oak was used (M+ toast) (kindly provided by Seguin Moreau), which was submerged in fortified wine used in Port wine production (kindly provided by Quinta do Portal S.A.) with contact conditions and wood and wine composition as described in Coelho, Teixeira, Domingues, Tavares, and Oliveira (2019). Prior to its application, wood was separated from the fortified wine and excess liquid was removed with sorbent paper.
For the extraction assay with different beverages a commercial red wine (12.5\% ethanol), a commercial lager beer ( $4.7 \%$ ethanol) and a craft grape marc spirit ( $41 \%$ ethanol) were used. For the formulation and sensory evaluation assays a pilsner craft beer (5\% ethanol) was used (kindly provided by Cerveja Letra).

\subsection{Wood contact with beverages}

Extractions were performed to assess impact of reused wood on the volatile fraction of different beverages. Used wood chips (cubes with 3 mm sides) were immersed in beer, wine and grape marc spirit at a proportion of $20 \mathrm{~g} / \mathrm{L}$. Contacts were conducted in Pyrex tubes $(16 \mathrm{~mm} \times$ $100 \mathrm{~mm}$, wall thickness $1.8 \mathrm{~mm}$ ) fitted with caps with Teflon membranes to prevent losses by evaporation. Tubes were placed horizontally in an incubator at $40^{\circ} \mathrm{C}$, with orbital agitation $\left(150 \mathrm{~min}^{-1}\right)$ during $48 \mathrm{~h}$. Also, for control purposes, beverages were placed in the same conditions without application of wood, in order to discriminate transformations caused only by temperature. After contact, beverages were separated from wood by decantation and further filtered with a $0.22 \mu \mathrm{m}$ cellulose acetate filter for analysis of volatile compounds. Beer (B), wine (W) and grape marc spirit $(S)$ were characterized in their initial state, after residence at $40{ }^{\circ} \mathrm{C}$ without wood (B40, W40, S40) and with wood (BW40, WW40, SW40).

\subsection{Beer formulations}

For sensory evaluation, various formulations were prepared by combining different wood concentrations and contact temperatures. Three different wood concentrations were used, $10 \mathrm{~g} / \mathrm{L}, 20 \mathrm{~g} / \mathrm{L}$ and 30 $\mathrm{g} / \mathrm{L}$ as well as three different contact temperatures, $30^{\circ} \mathrm{C}, 40^{\circ} \mathrm{C}$ and 50 ${ }^{\circ} \mathrm{C}$. Extractions were conducted in $100 \mathrm{~mL}$ airtight flasks fitted with rubber stoppers and aluminum seals, placed during $48 \mathrm{~h}$ in an incubator with orbital agitation $\left(150 \mathrm{~min}^{-1}\right)$. Beers were separated from wood by decantation followed by centrifugation at $2700 \mathrm{~g}$ during $15 \mathrm{~min}$ for removal of suspended particles. Samples were identified by a CxTy key, where $C x$ refers to wood concentration at $x \mathrm{~g} / \mathrm{L}$ and $T y$ refers to contact temperature performed at $y{ }^{\circ} \mathrm{C}$. Controls of beer incubated at the same temperatures without addition of wood were also performed, identified as $B T y$, where $y$ refers to contact temperature in ${ }^{\circ} \mathrm{C}$.

\subsection{Sensory evaluation of beer}

Sensory evaluation of the different beer formulations was performed by five trained panelists. The panel was constituted by one certified Beer Judge Certification Program (BJCP) judge, one certified beer Sommelier and 3 experienced wine and beer tasters. Blinded samples were presented in tasting glasses simultaneously, to allow comparison between the different samples. Scoring was performed using a tasting sheet with identification of visual, aroma and taste descriptors, using a quantitative scale from 1 to 10 , where 1 corresponds to very low and 10 to very high intensity. Results were processed in agreement with the standard ISO 11035 (International Organization for Standardization, 1994). Outliers were removed and geometric mean $(G M)$ was calculated for each descriptor in each sample according to Equation (1).

$G M / \%=\sqrt{I \times F} \times 100$.

Where $I$ refers to relative intensity, which is the sum of the intensities given by the panelists for a given descriptor, divided by the maximum possible intensity, and $F$ refers to the relative frequency, which is the number of times that the descriptor was mentioned divided by the maximum number of times that it could be mentioned.

\subsection{Analysis of volatile compounds}

Volatile compounds were analyzed by gas chromatography coupled 
with mass spectrometry (GC-MS), following the previously reported procedure (Coelho et al., 2020). Each $8 \mathrm{~mL}$ sample was extracted with $400 \mu \mathrm{L}$ of dichloromethane (SupraSolv for gas chromatography, Merck), after adding 4-nonanol as internal standard (3.2 $\mu \mathrm{g})$. Extractions were performed in Pyrex tubes fitted with Teflon caps, with stir bar agitation during $15 \mathrm{~min}$. Extracts were then recovered with a glass Pasteur pipette, dehydrated with anhydrous sodium sulfate and analyzed in a Varian 3800 gas chromatograph equipped with a 1079 injector and an ion-trap mass spectrometer Varian Saturn 2000. Each $1 \mu \mathrm{L}$ injection was made in splitless mode ( $30 \mathrm{~s})$ in a Sapiens-Wax MS column $(30 \mathrm{~m} \times 0.15$ $\mathrm{mm} ; 0.15 \mu \mathrm{m}$ film thickness, Teknokroma). Helium 49 (Praxair) at a constant flow of $1.3 \mathrm{~mL} / \mathrm{min}$ was used as a carrier gas. The detector was set to electronic impact mode with an ionization energy of $70 \mathrm{eV}$, a mass acquisition range $(\mathrm{m} / \mathrm{z})$ from 35 to 260 and a $610 \mathrm{~ms}$ acquisition interval. The oven temperature was initially set to $60^{\circ} \mathrm{C}$ for $2 \mathrm{~min}$ and then raised to $234{ }^{\circ} \mathrm{C}$ at a rate of $3 \mathrm{~K} / \mathrm{min}$, raised again to $260{ }^{\circ} \mathrm{C}$ at $5 \mathrm{~K} / \mathrm{min}$ and finally maintained at $260{ }^{\circ} \mathrm{C}$ for $10 \mathrm{~min}$. Injector temperature was set to $250{ }^{\circ} \mathrm{C}$ with a $30 \mathrm{~mL} / \mathrm{min}$ split flow and transfer line was maintained at $250{ }^{\circ} \mathrm{C}$. Compounds were identified using MS Workstation version 6.9 (Varian) software, by comparing mass spectra and retention indices with those of pure standards and quantified as 4-nonanol equivalents.

\subsection{Statistical analysis}

Statistically significant differences in concentrations of volatile compounds in the assay with different beverages were evaluated by a pairwise multiple $t$-test using the Prism 6 software (GraphPad software Inc.). For the assay with different beer formulations, statistically significant differences were determined by a non-parametric KruskallWallis analysis, with Conover-Iman multiple pairwise comparisons, using the XLStat software (Addinsoft). For assessment of correlations between sensory and chemical characterization a multivariate Principal Component Analysis (PCA) was performed using Statistica software (version 7, StatSoft Inc.)

\section{Results and discussion}

\subsection{Impact of used oak chips in the aromatic fraction of different beverages}

With the aim of determining their contribution in additive ageing, a preliminary assay was performed promoting contact of used oak chips with different alcoholic beverages, namely beer, red wine and grape marc spirit. Changes in the volatile composition of beverages was monitored by GC-MS with the results presented in Table 1 .

Statistically significant differences were evaluated by pairwise multiple $t$-test, without correction for multiple comparisons. An important initial observation is that low impact was observed for the residence of the studied beverages at $40{ }^{\circ} \mathrm{C}$ without wood, regarding their volatile composition. Statistically significant differences when comparing initial composition with composition after residence at $40{ }^{\circ} \mathrm{C}$ without wood were only found for 5-methylfurfural and benzaldehyde in beer, which were found in concentrations near the limit of detection and quantification; ethyl octanoate and ethyl decanoate in wine, which could arise from residual esterification of the corresponding acids with ethanol; and hexyl acetate, 2-phenylethanol, 4-ethylguaiacol and 4-ethylphenol in grape marc spirit, for which no significant trends or mechanisms could be identified. When applying used wood to beverages, several changes were observed. Despite having been previously in contact with fortified wine, used woods still had significant amount of wood compounds to be extracted, coherent with the previously reported (Coelho, Teixeira, et al., 2019). Characteristic wood extractives, namely volatile phenols and aldehydes, were transferred to the beverages by contact with wood, with statistically significant increases observed either in beer, wine and grape marc spirit. Increase in concentrations of furan compounds was only statistically significant for beer and wine, with variations in grape marc distillate masked by the initial high furan compounds concentrations.

Along with characteristic wood extractives, fortified wine compounds were also transferred to the beverages with the reuse of wood. As reported previously, characteristic wine esters (ethyl lactate, diethyl succinate and diethyl malate) were adsorbed by wood during the previous contact with fortified wine (Coelho, Teixeira, et al., 2019) and the reuse of wood in a subsequent ageing step led to their transference to the studied matrices. However, transference of these aroma compounds was more significant in the application of used wood to beer, where ethyl lactate, diethyl succinate and diethyl malate were absent. Also, for the specific case of diethyl malate, statistically significant increases were observed for application of wood to all the studied beverages. Extraction of additional wine volatiles adsorbed in wood, namely alcohols 3-methyl-1-butanol and 2-phenylethanol, monoethyl succinate and octanoic acid was not evident, probably hindered by the deviations in GC-MS analysis and the already high content of these compounds in the beverages. Apart from extraction of volatile compounds, compound losses inherent to subtractive ageing were also observed, as a direct result of application of wood.

Concentrations of ethyl octanoate and ethyl decanoate decreased significantly in all beverages put in contact with wood. Moreover, in grape marc spirit several esters were adsorbed, with decrease in concentrations of hexyl acetate, 2-phenylethyl acetate and ethyl dodecanoate. As stated previously, wood adsorbs several compounds from wine, mainly due to hydrophobic interactions (Coelho, Teixeira, et al., 2019) and these observations are corroborated by the results obtained in this work. Esters whose concentrations decreased due to wood contact, have high $x \log P 3$ values, as presented in Table 2, again reinforcing that their hydrophobic nature is the main driver of wood sorption. Significant decreases were also observed for decanoic acid concentration in beer and wine and nonanal in grape marc spirit, which have comparable XLogP3 values.

In a similar process, Sterckx and collaborators applied American oak wood chips for beer ageing, leaving wood in contact with beer during 60 $\mathrm{d}$ at $20^{\circ} \mathrm{C}$, following variation in concentration of volatile monophenols (Sterckx et al., 2012a; 2012b). These authors reported syringaldehyde concentrations of about $600 \mu \mathrm{g} / \mathrm{L}$, reached after $30 \mathrm{~d}$ of contact with medium toast unused American oak chips (Sterckx et al., 2012a), which corresponds roughly to an extraction of syringaldehyde, per mass of dry wood, of $120(\mu \mathrm{g} / \mathrm{L}) / \mathrm{g}$. Results presented in this work show a final syringaldehyde concentration of about $2500 \mu \mathrm{g} / \mathrm{L}$, attained after application of $20 \mathrm{~g} / \mathrm{L}$ of hydrated used oak wood, which accounts for an extraction of syringaldehyde, per mass of dry wood, of about 210 $(\mu \mathrm{g} / \mathrm{L}) / \mathrm{g}$. Sterckx also reported concentrations of vanillin of about 90 $\mu \mathrm{g} / \mathrm{L}$ (Sterckx, Saison, \& Delvaux, 2012b) accounting roughly for the extraction, per mass of dry wood, of $15(\mu \mathrm{g} / \mathrm{L}) / \mathrm{g}$, whereas in the presented work, the attained value was $38(\mu \mathrm{g} / \mathrm{L}) / \mathrm{g}$. These differences in extraction yields can derive from differences in oak wood composition, considering the reported variability within oak origin (Doussot, De Jéso, Quideau, \& Pardon, 2002). On the other hand, higher syringaldehyde and vanillin extraction concentrations, even from a previously used wood, can be a result of the higher contact temperature used, which was previously shown to influence significantly extraction of several characteristic wood compounds (Coelho, Teixeira, et al., 2019). Sterckx also reported an increase in $20 \mu \mathrm{g} / \mathrm{L}$ in eugenol concentration, accounting for about $4(\mu \mathrm{g} / \mathrm{L}) / \mathrm{g}$, whereas in the presented results an extraction of about $2(\mu \mathrm{g} / \mathrm{L}) / \mathrm{g}$ was obtained. As also demonstrated by Coelho, Domingues, Teixeira, Oliveira, and Tavares (2019), eugenol extraction is more influenced by ethanol concentration in the extractive matrix, which in our work was of $4.7 \%$, by volume, and in Sterckx and collaborators' work of $8.3 \%$, by volume, which in combination with depletion of eugenol in the preceding contact with wine justifies the observed differences. Lastly, Sterckx et al. (2012) also reported reduction in 4-vinylguaiacol concentration, similarly to the observed in this work. 
Table 1

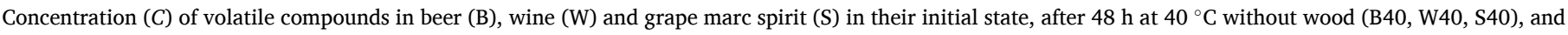
after $48 \mathrm{~h}$ at $40{ }^{\circ} \mathrm{C}$ with reused wood (BW40, WW40, SW40). Errors represent standard deviation of independent duplicates.

\begin{tabular}{|c|c|c|c|c|c|c|c|c|c|}
\hline Compound & B & B40 & BW40 & $\mathrm{W}$ & W40 & WW40 & $S$ & $\mathrm{~S} 40$ & SW 40 \\
\hline & $C /(\mu \mathrm{g} / \mathrm{L})$ & $C /(\mu \mathrm{g} / \mathrm{L})$ & $C /(\mu \mathrm{g} / \mathrm{L})$ & $C /(\mu \mathrm{g} / \mathrm{L})$ & $C /(\mu \mathrm{g} / \mathrm{L})$ & $C /(\mu \mathrm{g} / \mathrm{L})$ & $C /(\mu \mathrm{g} / \mathrm{L})$ & $C /(\mu \mathrm{g} / \mathrm{L})$ & $C /(\mu \mathrm{g} / \mathrm{L})$ \\
\hline \multicolumn{10}{|l|}{ Esters } \\
\hline isobutyl acetate & $18.7 \pm 3.6$ & $17.8 \pm 2.5$ & $19.0 \pm 4.9$ & - & - & - & $\begin{array}{l}813.7 \pm \\
102.6\end{array}$ & $844.7 \pm 4.7^{*}$ & $604.8 \pm 71.8^{*}$ \\
\hline ethyl butyrate & $41.1 \pm 0.1$ & $40.7 \pm 4.7$ & $46.8 \pm 9.5$ & $106.6 \pm 2.0$ & $100.0 \pm 2.8$ & $111.2 \pm 5.5$ & $\begin{array}{l}1605.2 \pm \\
106.6\end{array}$ & $\begin{array}{l}1677.0 \pm \\
43.9\end{array}$ & $\begin{array}{l}1566.0 \pm \\
171.0\end{array}$ \\
\hline ethyl 2-methylbutyrate & - & - & - & - & - & - & $771.1 \pm 87.5$ & $823.0 \pm 13.0$ & $740.0 \pm 91.3$ \\
\hline ethyl 3-methylbutyrate & - & - & - & - & - & - & $\begin{array}{l}2989.6 \pm \\
181.7\end{array}$ & $\begin{array}{l}3263.4 \pm \\
408.6\end{array}$ & $\begin{array}{l}3175.2 \pm \\
362.3\end{array}$ \\
\hline isoamyl acetate & $\begin{array}{l}988.0 \pm \\
9.3\end{array}$ & $\begin{array}{l}982.8 \pm \\
89.1\end{array}$ & $\begin{array}{l}1029.7 \pm \\
166.0\end{array}$ & $\begin{array}{l}580.1 \pm \\
35.9\end{array}$ & $604.3 \pm 6.4$ & $\begin{array}{l}556.3 \pm \\
65.2\end{array}$ & $\begin{array}{l}4703.7 \pm \\
48.1\end{array}$ & $\begin{array}{l}5001.5 \pm \\
212.6\end{array}$ & $\begin{array}{l}4690.9 \pm \\
490.7\end{array}$ \\
\hline ethyl pentanoate & - & - & - & - & - & - & $167.3 \pm 16.3$ & $217.1 \pm 5.5$ & $173.5 \pm 25.6$ \\
\hline ethyl 2-butenoate & - & - & - & - & - & - & $570.8 \pm 18.7$ & $580.5 \pm 21.8$ & $550.9 \pm 55.0$ \\
\hline ethyl hexanoate & $\begin{array}{l}137.9 \pm \\
0.2\end{array}$ & $\begin{array}{l}140.7 \pm \\
13.0\end{array}$ & $\begin{array}{l}126.6 \pm \\
22.5\end{array}$ & $\begin{array}{l}257.5 \pm \\
11.8\end{array}$ & $\begin{array}{l}288.3 \pm \\
10.0\end{array}$ & $\begin{array}{l}235.6 \pm \\
22.0\end{array}$ & $\begin{array}{l}6393.0 \pm \\
309.9\end{array}$ & $\begin{array}{l}6867.9 \pm \\
422.3\end{array}$ & $\begin{array}{l}5964.1 \pm \\
632.6\end{array}$ \\
\hline hexyl acetate & - & - & - & - & - & - & $247.8 \pm 6.8$ & $268.7 \pm 0.4^{*}$ & $208.8 \pm 19.8^{*}$ \\
\hline ethyl heptanoate & - & - & - & - & - & - & $569.2 \pm 20.2$ & $550.8 \pm 8.8$ & $474.6 \pm 36.5$ \\
\hline ethyl lactate & - & - & $\begin{array}{l}59.0 \pm \\
16.3^{*}\end{array}$ & $\begin{array}{l}3454.7 \pm \\
937.4\end{array}$ & $\begin{array}{l}3576.2 \pm \\
161.5\end{array}$ & $\begin{array}{l}3907.3 \pm \\
925.5\end{array}$ & $\begin{array}{l}1297.2 \pm \\
98.4\end{array}$ & $1411.6 \pm 2.1$ & $\begin{array}{l}1660.5 \pm \\
284.1\end{array}$ \\
\hline ethyl 2-hexenoate & - & - & - & - & - & - & $365.5 \pm 10.6$ & $358.3 \pm 13.3$ & $361.7 \pm 41.1$ \\
\hline ethyl octanoate & $\begin{array}{l}129.0 \pm \\
4.4\end{array}$ & $\begin{array}{l}133.4 \pm \\
6.6^{*}\end{array}$ & $\begin{array}{l}68.0 \pm \\
13.4^{*}\end{array}$ & $\begin{array}{l}237.5 \pm \\
13.7\end{array}$ & $\begin{array}{l}316.3 \pm \\
12.2^{*}\end{array}$ & $\begin{array}{l}152.8 \pm \\
12.2^{*}\end{array}$ & $\begin{array}{l}24531.9 \pm \\
534.8\end{array}$ & $\begin{array}{l}26831.5 \pm \\
1570.6^{*}\end{array}$ & $\begin{array}{l}20902.4 \pm \\
1731.3^{*}\end{array}$ \\
\hline ethyl-3-hydroxybutyrate & - & - & - & $74.2 \pm 17.0$ & $78.4 \pm 4.6$ & $93.1 \pm 17.2$ & - & - & - \\
\hline ethyl decanoate & $18.6 \pm 0.8$ & $20.7 \pm 2.4^{*}$ & $11.1 \pm 3.1^{*}$ & $30.0 \pm 0.9$ & $33.7 \pm 0.3^{*}$ & $11.7 \pm 0.3^{*}$ & $\begin{array}{l}25557.4 \pm \\
285.6\end{array}$ & $\begin{array}{l}32014.4 \pm \\
6534.6^{*}\end{array}$ & $\begin{array}{l}11350.1 \pm \\
796.2^{*}\end{array}$ \\
\hline diethyl succinate & - & - & $\begin{array}{l}666.1 \pm \\
123.7^{*}\end{array}$ & $\begin{array}{l}7562.9 \pm \\
913.9\end{array}$ & $\begin{array}{l}7296.9 \pm \\
29.3\end{array}$ & $\begin{array}{l}8087.6 \pm \\
642.2\end{array}$ & $\begin{array}{l}8252.1 \pm \\
106.3\end{array}$ & $\begin{array}{l}8317.3 \pm \\
206.9\end{array}$ & $\begin{array}{l}8551.1 \pm \\
694.2\end{array}$ \\
\hline methyl salycilate & - & - & - & - & - & - & $\begin{array}{l}1167.4 \pm \\
27.4\end{array}$ & $\begin{array}{l}1144.5 \pm \\
23.4\end{array}$ & $1058.7 \pm 62.3$ \\
\hline ethyl phenylacetate & - & - & - & - & - & - & $624.2 \pm 27.4$ & $601.0 \pm 0.8$ & $577.3 \pm 29.2$ \\
\hline 2-phenylethyl acetate & $\begin{array}{l}788.9 \pm \\
15.2\end{array}$ & $\begin{array}{l}809.4 \pm \\
68.0\end{array}$ & $\begin{array}{l}735.0 \pm \\
141.0\end{array}$ & $\begin{array}{l}128.4 \pm \\
16.0\end{array}$ & $\begin{array}{l}119.6 \pm \\
10.0\end{array}$ & $106.1 \pm 7.7$ & $904.2 \pm 19.0$ & $913.4 \pm 15.5^{*}$ & $752.3 \pm 34.3^{*}$ \\
\hline ethyl dodecanoate & - & - & - & - & - & - & $\begin{array}{l}8144.5 \pm \\
45.8\end{array}$ & $\begin{array}{l}13516.4 \pm \\
4377.1^{*}\end{array}$ & $\begin{array}{l}1857.1 \pm \\
90.8^{*}\end{array}$ \\
\hline diethyl malate & - & - & $35.3 \pm 8.2^{*}$ & $\begin{array}{l}151.4 \pm \\
34.6\end{array}$ & $\begin{array}{l}150.2 \pm \\
5.3^{*}\end{array}$ & $\begin{array}{l}200.6 \pm \\
20.2^{*}\end{array}$ & - & - & $54.8 \pm 11.4^{*}$ \\
\hline ethyl cinnamate & - & - & - & - & - & - & $282.8 \pm 11.2$ & $260.4 \pm 5.3$ & $238.7 \pm 13.9$ \\
\hline \multicolumn{10}{|l|}{ Alcohols } \\
\hline 2-methyl-1-propanol & $\begin{array}{l}140.7 \pm \\
14.5\end{array}$ & $\begin{array}{l}139.5 \pm \\
50.4\end{array}$ & $\begin{array}{l}198.5 \pm \\
58.1\end{array}$ & $\begin{array}{l}749.1 \pm \\
89.8\end{array}$ & $\begin{array}{l}869.3 \pm \\
73.0\end{array}$ & $\begin{array}{l}821.5 \pm \\
150.1\end{array}$ & $\begin{array}{l}5820.2 \pm \\
205.8\end{array}$ & $\begin{array}{l}5929.5 \pm \\
923.9\end{array}$ & $\begin{array}{l}5984.5 \pm \\
675.3\end{array}$ \\
\hline $\begin{array}{l}\text { 2-methyl-1-butanol+ 3- } \\
\text { methyl-1-butanol }\end{array}$ & $\begin{array}{l}5781.5 \pm \\
280.5\end{array}$ & $\begin{array}{l}5787.7 \pm \\
1594.4\end{array}$ & $\begin{array}{l}7796.4 \pm \\
2045.1\end{array}$ & $\begin{array}{l}20560.5 \pm \\
2889.4\end{array}$ & $\begin{array}{l}23359.9 \pm \\
57.9\end{array}$ & $\begin{array}{l}23400.2 \pm \\
5011.6\end{array}$ & $\begin{array}{l}103415.4 \pm \\
8988.9\end{array}$ & $\begin{array}{l}116358.1 \pm \\
1318.9\end{array}$ & $\begin{array}{l}128263.2 \pm \\
19837.8\end{array}$ \\
\hline 1-hexanol & - & - & - & $\begin{array}{l}533.3 \pm \\
67.1\end{array}$ & $\begin{array}{l}539.8 \pm \\
20.3\end{array}$ & $\begin{array}{l}591.1 \pm \\
101.8\end{array}$ & $\begin{array}{l}13181.2 \pm \\
1015.3\end{array}$ & $\begin{array}{l}14152.3 \pm \\
196.8\end{array}$ & $\begin{array}{l}14653.3 \pm \\
1890.5\end{array}$ \\
\hline Z-3-hexenol & - & - & - & $15.0 \pm 2.4$ & $14.5 \pm 0.5$ & $16.6 \pm 2.7$ & $330.9 \pm 25.1$ & $367.4 \pm 11.3$ & $384.7 \pm 55.8$ \\
\hline E-3-hexenol & - & - & - & $39.0 \pm 7.1$ & $39.6 \pm 0.2$ & $45.1 \pm 9.5$ & $580.8 \pm 49.0$ & $620.6 \pm 18.1$ & $639.9 \pm 80.8$ \\
\hline 1-octanol & $6.9 \pm 0.1$ & $7.4 \pm 0.9$ & $8.6 \pm 1.3$ & $18.4 \pm 1.9$ & $17.2 \pm 0.9$ & $16.9 \pm 2.5$ & $1481.4 \pm 4.4$ & $\begin{array}{l}1478.1 \pm \\
85.5\end{array}$ & $\begin{array}{l}1414.6 \pm \\
129.3\end{array}$ \\
\hline benzyl alcohol & $11.1 \pm 1.2$ & $13.1 \pm 4.5$ & $17.8 \pm 4.7$ & $\begin{array}{l}154.0 \pm \\
91.1\end{array}$ & $104.2 \pm 4.8$ & $\begin{array}{l}124.5 \pm \\
36.1\end{array}$ & $228.1 \pm 17.9$ & $231.1 \pm 19.5$ & $281.1 \pm 26.1$ \\
\hline 2-phenylethanol & $\begin{array}{l}6027.0 \pm \\
315.6\end{array}$ & $\begin{array}{l}6477.9 \pm \\
1480.8\end{array}$ & $\begin{array}{l}7893.9 \pm \\
1868.2\end{array}$ & $\begin{array}{l}9337.1 \pm \\
1869.4\end{array}$ & $\begin{array}{l}9286.7 \pm \\
99.0\end{array}$ & $\begin{array}{l}9969.5 \pm \\
1515.8\end{array}$ & $\begin{array}{l}9763.2 \pm \\
43.8\end{array}$ & $\begin{array}{l}10462.5 \pm \\
211.8\end{array}$ & $\begin{array}{l}11003.2 \pm \\
1480.6\end{array}$ \\
\hline \multicolumn{10}{|l|}{ Monoterpenic alcohols } \\
\hline Linalool & $8.9 \pm 0.3$ & $9.9 \pm 0.5$ & $9.5 \pm 1.8$ & $11.7 \pm 2.0$ & $10.4 \pm 0.4^{*}$ & $8.8 \pm 0.5^{*}$ & $391.6 \pm 10.0$ & $391.3 \pm 8.6$ & $368.3 \pm 28.5$ \\
\hline$\alpha$-terpineol & $5.6 \pm 0.1$ & $7.4 \pm 1.0$ & $7.6 \pm 1.4$ & $34.9 \pm 5.6$ & $33.1 \pm 0.4$ & $30.0 \pm 1.9$ & $861.6 \pm 17.7$ & $889.9 \pm 13.3$ & $864.0 \pm 44.8$ \\
\hline$\beta$-citronellol & $3.0 \pm 0.1$ & $3.4 \pm 0.5$ & $3.7 \pm 0.8$ & - & - & - & $558.7 \pm 35.8$ & $540.2 \pm 16.8$ & $503.0 \pm 39.3$ \\
\hline cis-nerolidol & - & - & - & - & - & - & $377.3 \pm 7.8$ & $355.9 \pm 42.0$ & $315.3 \pm 1.4$ \\
\hline \multicolumn{10}{|l|}{ Acids } \\
\hline acetic acid & $40.0 \pm 9.0$ & $\begin{array}{l}83.4 \pm \\
77.3\end{array}$ & $\begin{array}{l}46.6 \pm \\
13.5\end{array}$ & $\begin{array}{l}303.4 \pm \\
118.5\end{array}$ & $\begin{array}{l}298.6 \pm \\
48.4\end{array}$ & $\begin{array}{l}305.0 \pm \\
89.2\end{array}$ & - & - & - \\
\hline propanoic acid & $12.0 \pm 0.1$ & $15.0 \pm 3.7$ & $7.6 \pm 2.5$ & $47.3 \pm 33.6$ & $46.3 \pm 14.9$ & $25.6 \pm 10.6$ & - & - & - \\
\hline butanoic acid & $3.3 \pm 1.2$ & $5.8 \pm 2.4$ & $4.0 \pm 1.5$ & $29.4 \pm 14.1$ & $23.6 \pm 0.6$ & $16.8 \pm 5.5$ & - & - & - \\
\hline isovaleric acid & $17.8 \pm 2.1$ & $20.6 \pm 9.0$ & $21.5 \pm 5.9$ & $91.9 \pm 25.5$ & $93.9 \pm 1.3$ & $92.8 \pm 25.3$ & $\begin{array}{l}1272.2 \pm \\
73.2\end{array}$ & $\begin{array}{l}1345.5 \pm \\
136.1\end{array}$ & $\begin{array}{l}1505.3 \pm \\
207.7\end{array}$ \\
\hline hexanoic acid & $\begin{array}{l}295.1 \pm \\
20.7\end{array}$ & $\begin{array}{l}314.4 \pm \\
94.4\end{array}$ & $\begin{array}{l}327.0 \pm \\
83.1\end{array}$ & $\begin{array}{l}742.8 \pm \\
191.2\end{array}$ & $\begin{array}{l}660.2 \pm \\
44.8\end{array}$ & $\begin{array}{l}742.6 \pm \\
127.9\end{array}$ & $\begin{array}{l}3721.9 \pm \\
2867.8\end{array}$ & $\begin{array}{l}1833.9 \pm \\
79.2\end{array}$ & $\begin{array}{l}1976.4 \pm \\
318.7\end{array}$ \\
\hline octanoic acid & $\begin{array}{l}1896.3 \pm \\
74.6\end{array}$ & $\begin{array}{l}2040.3 \pm \\
244.4\end{array}$ & $\begin{array}{l}2182.2 \pm \\
408.4\end{array}$ & $\begin{array}{l}1606.8 \pm \\
198.0\end{array}$ & $\begin{array}{l}1555.1 \pm \\
0.3\end{array}$ & $\begin{array}{l}1574.4 \pm \\
131.8\end{array}$ & $\begin{array}{l}13013.4 \pm \\
197.0\end{array}$ & $\begin{array}{l}14085.0 \pm \\
1057.2\end{array}$ & $\begin{array}{l}12475.1 \pm \\
1241.8\end{array}$ \\
\hline
\end{tabular}

decanoic acid 
Table 1 (continued)

\begin{tabular}{|c|c|c|c|c|c|c|c|c|c|}
\hline Compound & B & B40 & BW40 & $\mathrm{W}$ & W40 & WW40 & $S$ & S40 & SW 40 \\
\hline & $\begin{array}{l}444.8 \pm \\
121.2\end{array}$ & $\begin{array}{l}462.8 \pm \\
68.5^{*}\end{array}$ & $\begin{array}{l}257.7 \pm \\
59.0^{*}\end{array}$ & $\begin{array}{l}466.8 \pm \\
42.0\end{array}$ & $\begin{array}{l}460.1 \pm \\
42.3^{*}\end{array}$ & $\begin{array}{l}292.2 \pm \\
13.6^{*}\end{array}$ & $\begin{array}{l}17977.0 \pm \\
118.4\end{array}$ & $\begin{array}{l}16888.1 \pm \\
711.9\end{array}$ & $\begin{array}{l}17033.0 \pm \\
1611.5\end{array}$ \\
\hline dodecanoic acid & - & - & - & - & - & - & $\begin{array}{l}5008.1 \pm \\
71.3\end{array}$ & $\begin{array}{l}5176.7 \pm \\
869.5\end{array}$ & $\begin{array}{l}4039.6 \pm \\
320.8\end{array}$ \\
\hline \multicolumn{10}{|l|}{ Furan compounds } \\
\hline Furfural & $84.7 \pm 4.5$ & $\begin{array}{l}102.9 \pm \\
45.5^{*}\end{array}$ & $\begin{array}{l}342.3 \pm \\
77.8^{*}\end{array}$ & $76.0 \pm 15.0$ & $\begin{array}{l}96.8 \pm \\
17.8^{*}\end{array}$ & $\begin{array}{l}320.6 \pm \\
56.6^{*}\end{array}$ & $\begin{array}{l}4207.7 \pm \\
64.7\end{array}$ & $\begin{array}{l}4518.0 \pm \\
106.8\end{array}$ & $\begin{array}{l}4894.0 \pm \\
682.3\end{array}$ \\
\hline linalool furanic oxide & - & - & - & - & - & - & $313.1 \pm 3.9$ & $316.9 \pm 14.5$ & $319.0 \pm 35.1$ \\
\hline 5-methylfurfural & - & $1.5 \pm 0.5^{*}$ & $\begin{array}{l}109.7 \pm \\
21.9^{*}\end{array}$ & - & - & $\begin{array}{l}133.6 \pm \\
17.5^{*}\end{array}$ & $678.8 \pm 49.8$ & $719.4 \pm 16.5$ & $783.5 \pm 90.1$ \\
\hline 5-hydroxymethylfurfural & $13.1 \pm 2.5$ & $16.7 \pm 8.6$ & $24.6 \pm 9.4$ & - & - & - & - & - & - \\
\hline \multicolumn{10}{|l|}{ Lactones } \\
\hline cis-oak lactone & - & - & $\begin{array}{l}364.2 \pm \\
66.3^{*}\end{array}$ & - & - & $\begin{array}{l}359.4 \pm \\
22.7^{*}\end{array}$ & - & - & $548.6 \pm 53.1^{*}$ \\
\hline trans-oak lactone & - & - & $\begin{array}{l}138.2 \pm \\
24.6^{*}\end{array}$ & - & - & $144.1 \pm 6.4^{*}$ & - & - & $163.3 \pm 11.4^{*}$ \\
\hline$\gamma$-nonalactone & $38.3 \pm 1.2$ & $42.3 \pm 4.0$ & $39.2 \pm 7.2$ & $11.5 \pm 2.8$ & $10.6 \pm 0.0$ & $10.6 \pm 0.3$ & $233.8 \pm 10.6$ & $231.6 \pm 8.8$ & $210.3 \pm 6.5$ \\
\hline $\begin{array}{c}\gamma \text {-ethoxycarbonyl- } \\
\gamma \text {-butyrolactone }\end{array}$ & - & - & - & $\begin{array}{l}404.1 \pm \\
55.6\end{array}$ & $\begin{array}{l}451.1 \pm \\
20.1\end{array}$ & $\begin{array}{l}513.6 \pm \\
31.2\end{array}$ & - & - & - \\
\hline \multicolumn{10}{|l|}{ Aldehydes } \\
\hline benzaldehyde & - & $5.1 \pm 0.6^{*}$ & $16.3 \pm 2.9^{*}$ & - & - & - & $\begin{array}{l}2337.5 \pm \\
16.8\end{array}$ & $\begin{array}{l}2427.9 \pm \\
55.0\end{array}$ & $\begin{array}{l}2295.7 \pm \\
212.0\end{array}$ \\
\hline vanillin & $15.6 \pm 0.1$ & $14.7 \pm 4.7^{*}$ & $\begin{array}{l}459.0 \pm \\
119.8^{*}\end{array}$ & - & - & $\begin{array}{l}480.8 \pm \\
22.6^{*}\end{array}$ & - & - & $542.1 \pm 93.7^{*}$ \\
\hline syringaldehyde & - & - & $\begin{array}{l}2513.2 \pm \\
707.3^{*}\end{array}$ & $\begin{array}{l}147.3 \pm \\
57.8\end{array}$ & $\begin{array}{l}317.0 \pm \\
58.4^{*}\end{array}$ & $\begin{array}{l}2573.9 \pm \\
169.5^{*}\end{array}$ & - & - & $\begin{array}{l}2812.3 \pm \\
92.0^{*}\end{array}$ \\
\hline sinapaldehyde & - & - & $\begin{array}{l}2377.4 \pm \\
761.7^{*}\end{array}$ & - & - & $\begin{array}{l}2258.2 \pm \\
636.4^{*}\end{array}$ & - & - & $\begin{array}{l}4012.7 \pm \\
569.8^{*}\end{array}$ \\
\hline octanal & - & - & - & - & - & - & $178.1 \pm 4.5$ & $188.4 \pm 0.7^{*}$ & $155.7 \pm 11.7^{*}$ \\
\hline nonanal & - & - & - & - & - & - & $1428.6 \pm 2.0$ & $\begin{array}{l}1544.6 \pm \\
40.0\end{array}$ & $1425.1 \pm 86.6$ \\
\hline \multicolumn{10}{|l|}{ Volatile phenols } \\
\hline 4-methylguaiacol & - & - & $18.7 \pm 3.4^{*}$ & - & - & - & - & - & $68.6 \pm 6.4^{*}$ \\
\hline 4-ethylguaiacol & $0.8 \pm 0.0$ & $0.6 \pm 0.9^{*}$ & $4.4 \pm 0.9^{*}$ & - & - & - & $229.2 \pm 2.6$ & $263.2 \pm 10.0$ & $263.4 \pm 11.4$ \\
\hline eugenol & - & - & $17.6 \pm 3.3^{*}$ & $1.3 \pm 0.5$ & $5.6 \pm 5.8$ & $14.1 \pm 1.2$ & $456.2 \pm 0.9$ & $423.6 \pm 11.5$ & $422.1 \pm 16.6$ \\
\hline 4-ethylphenol & - & - & - & - & - & - & $281.8 \pm 4.8$ & $236.4 \pm 0.0$ & $249.4 \pm 27.1$ \\
\hline 4-vinylguaiacol & $\begin{array}{l}94.8 \pm \\
63.6\end{array}$ & $\begin{array}{l}104.3 \pm \\
24.7^{*}\end{array}$ & $38.2 \pm 8.0^{*}$ & - & - & - & - & - & - \\
\hline 2,6-dimethoxyphenol & - & - & $\begin{array}{l}71.4 \pm \\
16.8^{*}\end{array}$ & $31.5 \pm 9.5$ & $32.5 \pm 3.3^{*}$ & $112.3 \pm 1.2^{*}$ & - & - & - \\
\hline 4-vinylphenol & $11.2 \pm 0.2$ & $16.2 \pm 4.1^{*}$ & $5.5 \pm 1.7^{*}$ & - & - & - & - & - & - \\
\hline acetovanillone & $8.3 \pm 0.0$ & $11.3 \pm 3.5^{*}$ & $\begin{array}{l}90.9 \pm \\
22.9^{*}\end{array}$ & - & - & - & - & - & - \\
\hline tyrosol & $22.1 \pm 5.2$ & $\begin{array}{l}47.1 \pm \\
31.7\end{array}$ & $\begin{array}{l}43.4 \pm \\
17.5\end{array}$ & - & - & - & - & - & - \\
\hline \multicolumn{10}{|l|}{ Sulphur compounds } \\
\hline methionol & - & $24.4 \pm 9.4$ & $17.6 \pm 5.0$ & $\begin{array}{l}131.9 \pm \\
36.0\end{array}$ & $\begin{array}{l}124.9 \pm \\
11.7\end{array}$ & $96.8 \pm 20.6$ & - & - & - \\
\hline \multicolumn{10}{|l|}{ C13-norisoprenoids } \\
\hline$\beta$-damascenone & - & $1.9 \pm 1.3$ & $4.0 \pm 1.4$ & - & - & - & - & - & - \\
\hline 3-hydroxy- $\beta$-damascone & $15.0 \pm 1.2$ & $18.9 \pm 3.3$ & $15.3 \pm 4.6$ & - & - & - & - & - & - \\
\hline 3-hydroxy- $\beta$-ionone & $5.6 \pm 0.1$ & $8.3 \pm 1.5$ & $13.0 \pm 2.6$ & - & - & - & - & - & - \\
\hline \multicolumn{10}{|l|}{ Other } \\
\hline acetoin & - & - & - & $\begin{array}{l}143.1 \pm \\
48.0\end{array}$ & $153.9 \pm 5.3$ & $\begin{array}{l}141.2 \pm \\
28.9\end{array}$ & - & - & - \\
\hline
\end{tabular}

- not detected

Table 2

XLogP3-AA values reported for the volatile compounds adsorbed in wood (National Center for Biotechnology Information, 2020).

\begin{tabular}{llll}
\hline Compound & XLogP3-AA & Compound & XLogP3-AA \\
\hline 2-phenylethyl acetate & 2.3 & decanoic acid & 4.1 \\
hexyl acetate & 2.4 & ethyl decanoate & 4.6 \\
nonanal & 3.3 & ethyl dodecanoate & 5.6 \\
\hline
\end{tabular}

In similar works focusing wine, Bautista-Ortín and collaborators have studied the application of oak alternatives for the ageing of wine, submerging American oak shavings in red wine in stainless steel tanks using a $2.6 \mathrm{~g} / \mathrm{L}$ proportion (Bautista-Ortín et al., 2008). The authors reported maximum concentrations of furfural of $326 \mu \mathrm{g} / \mathrm{L}$ accounting for an extraction, per mass of wood, of $125(\mu \mathrm{g} / \mathrm{L}) / \mathrm{g}$, and of 5-methylfurfural of $37 \mu \mathrm{g} / \mathrm{L}$ accounting for extraction of $14(\mu \mathrm{g} / \mathrm{L}) / \mathrm{g}$ (Bautista-Ortín et al., 2008). In the presented results similar concentrations of furfural (about $320 \mu \mathrm{g} / \mathrm{L}$ ) and higher concentrations of 5-methylfurfural (133 $\mu \mathrm{g} / \mathrm{L})$ were attained with previously used oak chips. Lower extraction 
Table 3

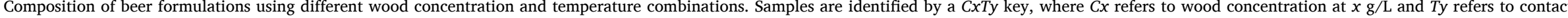

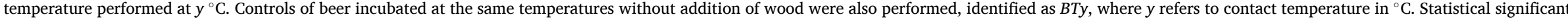
similarities/differences are marked by a to $\mathrm{h}$ letters. Errors represent standard deviation of independent duplicates.

\begin{tabular}{|c|c|c|c|c|c|c|c|c|c|c|c|c|c|}
\hline & Compound & BT30 & С10T30 & С20T30 & Сз0Т30 & BT40 & C10T40 & C20T40 & C30T40 & BT50 & C10T50 & C20T50 & C30T50 \\
\hline & & $C /(\mu \mathrm{g} / \mathrm{L})$ & $C /(\mu \mathrm{g} / \mathrm{L})$ & $C /(\mu \mathrm{g} / \mathrm{L})$ & $C /(\mu \mathrm{g} / \mathrm{L})$ & $C /(\mu \mathrm{g} / \mathrm{L})$ & $C /(\mu \mathrm{g} / \mathrm{L})$ & $C /(\mu \mathrm{g} / \mathrm{L})$ & $C /(\mu \mathrm{g} / \mathrm{L})$ & $C /(\mu \mathrm{g} / \mathrm{L})$ & $C /(\mu \mathrm{g} / \mathrm{L})$ & $C /(\mu \mathrm{g} / \mathrm{L})$ & $C /(\mu \mathrm{g} / \mathrm{L})$ \\
\hline & Esters & & & & & & & & & & & & \\
\hline 1 & isoamyl acetate & $\begin{array}{l}842.7 \pm \\
9.8^{\mathrm{bc}}\end{array}$ & $\begin{array}{l}753.2 \pm \\
11.3^{\mathrm{abc}}\end{array}$ & $\begin{array}{l}723.4 \pm \\
43.0^{\mathrm{abc}}\end{array}$ & $\begin{array}{l}682.1 \pm \\
36.3^{\mathrm{a}}\end{array}$ & $\begin{array}{l}947.8 \pm \\
119.6^{\mathrm{bc}}\end{array}$ & $\begin{array}{l}795.2 \pm \\
47.2^{\mathrm{abc}}\end{array}$ & $\begin{array}{l}730.1 \pm \\
0.3^{\mathrm{abc}}\end{array}$ & $\begin{array}{l}826.7 \pm \\
182.8^{\text {abc }}\end{array}$ & $\begin{array}{l}896.9 \pm \\
38.7^{c}\end{array}$ & $\begin{array}{l}746.9 \pm \\
25.2^{\mathrm{abc}}\end{array}$ & $\begin{array}{l}744.2 \pm \\
48.6^{\mathrm{abc}}\end{array}$ & $\begin{array}{l}723.7 \pm \\
7.4^{\mathrm{ab}}\end{array}$ \\
\hline 2 & ethyl hexanoate & $89.0 \pm 3.2^{\mathrm{bc}}$ & $77.2 \pm 1.8^{\mathrm{ab}}$ & $83.1 \pm 4.2^{\mathrm{abc}}$ & $74.4 \pm 5.1^{\mathrm{a}}$ & $\begin{array}{l}105.3 \pm \\
14.7^{\mathrm{c}}\end{array}$ & $85.3 \pm 4.8^{\mathrm{abc}}$ & $\begin{array}{l}83.4 \pm \\
0.1^{\mathrm{abc}}\end{array}$ & $\begin{array}{l}95.4 \pm \\
19.0^{\mathrm{abc}}\end{array}$ & $90.3 \pm 6.4^{\mathrm{bc}}$ & $75.1 \pm 4.5^{\mathrm{ab}}$ & $81.7 \pm 3.1^{\mathrm{abc}}$ & $74.9 \pm 1.3^{\mathrm{a}}$ \\
\hline 3 & ethyl lactate & - & $\begin{array}{l}160.1 \pm \\
24.5^{\mathrm{ab}}\end{array}$ & $278.2 \pm 23.7^{\mathrm{d}}$ & $\begin{array}{l}314.1 \pm \\
20.6^{\mathrm{de}}\end{array}$ & - & $\begin{array}{l}142.9 \pm \\
0.2^{\mathrm{ab}}\end{array}$ & $\begin{array}{l}209.6 \pm \\
7.0^{\mathrm{bc}}\end{array}$ & $\begin{array}{l}494.9 \pm \\
142.6^{\mathrm{e}}\end{array}$ & - & $117.7 \pm 3.6^{\mathrm{a}}$ & $\begin{array}{l}245.7 \pm \\
27.5^{\mathrm{cd}}\end{array}$ & $\begin{array}{l}367.8 \pm \\
51.8^{\mathrm{e}}\end{array}$ \\
\hline 4 & ethyl octanoate & $462.9 \pm 9.7^{\mathrm{ef}}$ & $\begin{array}{l}396.9 \pm \\
8.0^{\text {cde }}\end{array}$ & $\begin{array}{l}410.6 \pm \\
20.1^{\text {def }}\end{array}$ & $\begin{array}{l}354.2 \pm \\
31.9^{\text {bed }}\end{array}$ & $\begin{array}{l}571.6 \pm \\
110.6^{\mathrm{f}}\end{array}$ & $\begin{array}{l}467.8 \pm \\
23.6^{\mathrm{ef}}\end{array}$ & $\begin{array}{l}398.8 \pm \\
27.9^{\text {cde }}\end{array}$ & $\begin{array}{l}436.3 \pm \\
127.6^{\text {def }}\end{array}$ & $\begin{array}{l}172.8 \pm \\
4.0^{\mathrm{abc}}\end{array}$ & $\begin{array}{l}145.0 \pm \\
8.8^{\mathrm{ab}}\end{array}$ & $145.1 \pm 4.2^{\mathrm{ab}}$ & $122.2 \pm 3.8^{\mathrm{a}}$ \\
\hline 5 & ethyl decanoate & $149.5 \pm 0.3^{\mathrm{d}}$ & $105.6 \pm 5.2^{\mathrm{c}}$ & $96.0 \pm 9.2^{c}$ & $70.4 \pm 5.3^{\mathrm{b}}$ & $\begin{array}{l}234.4 \pm \\
40.5^{\mathrm{d}}\end{array}$ & $\begin{array}{l}157.4 \pm \\
11.4^{\mathrm{d}}\end{array}$ & $111.8 \pm 6.5^{\mathrm{c}}$ & $108.3 \pm 31.1^{\mathrm{c}}$ & $71.3 \pm 0.0^{\mathrm{b}}$ & $50.2 \pm 3.5^{\mathrm{ab}}$ & $44.4 \pm 1.8^{\mathrm{a}}$ & $32.5 \pm 2.3^{\mathrm{a}}$ \\
\hline 6 & diethyl succinate & - & $190.8 \pm 4.9^{\mathrm{a}}$ & $\begin{array}{l}415.1 \pm \\
23.2^{\text {cd }}\end{array}$ & $\begin{array}{l}558.2 \pm \\
18.8^{\mathrm{de}}\end{array}$ & - & $\begin{array}{l}199.4 \pm \\
8.2^{\mathrm{ab}}\end{array}$ & $\begin{array}{l}392.5 \pm \\
2.2^{\mathrm{bc}}\end{array}$ & $\begin{array}{l}689.4 \pm \\
148.7^{\mathrm{e}}\end{array}$ & - & $180.3 \pm 1.9^{\mathrm{a}}$ & $\begin{array}{l}395.9 \pm \\
19.77^{\mathrm{bc}}\end{array}$ & $\begin{array}{l}573.5 \pm \\
32.9^{\mathrm{e}}\end{array}$ \\
\hline 7 & 2-phenylethyl acetate & $371.5 \pm 1.1^{\mathrm{d}}$ & $\begin{array}{l}296.0 \pm \\
1.0^{\mathrm{bc}}\end{array}$ & $\begin{array}{l}269.2 \pm \\
13.5^{\mathrm{ab}}\end{array}$ & $\begin{array}{l}214.4 \pm \\
12.8^{\mathrm{a}}\end{array}$ & $\begin{array}{l}409.8 \pm \\
61.8^{\mathrm{d}}\end{array}$ & $\begin{array}{l}318.5 \pm \\
15.0^{\text {cd }}\end{array}$ & $\begin{array}{l}267.4 \pm \\
2.9^{\text {ab }}\end{array}$ & $\begin{array}{l}272.5 \pm \\
58.8^{\text {abc }}\end{array}$ & $370.9 \pm 6.2^{\mathrm{d}}$ & $\begin{array}{l}296.2 \pm \\
1.6^{\mathrm{bc}}\end{array}$ & $\begin{array}{l}278.4 \pm \\
12.3^{\text {abc }}\end{array}$ & $245.7 \pm 9.4^{\mathrm{a}}$ \\
\hline \multirow[t]{2}{*}{8} & diethyl malate & - & $9.4 \pm 0.4^{\mathrm{a}}$ & $17.9 \pm 1.7^{\mathrm{bc}}$ & $24.5 \pm 0.3^{\mathrm{cd}}$ & - & $9.2 \pm 0.1^{\mathrm{a}}$ & $15.4 \pm 0.4^{\mathrm{b}}$ & $33.4 \pm 7.7^{\mathrm{d}}$ & - & $9.2 \pm 1.6^{\mathrm{a}}$ & $16.7 \pm 2.0^{\mathrm{b}}$ & $25.4 \pm 2.5^{\mathrm{cd}}$ \\
\hline & Alcohols & & & & & & & & & & & & \\
\hline 9 & 2-methyl-1-propanol & $\begin{array}{l}1182.5 \pm \\
361.3^{\text {bcd }}\end{array}$ & $\begin{array}{l}1209.7 \pm \\
353.3^{\text {bcd }}\end{array}$ & $\begin{array}{l}1070.5 \pm \\
10.9^{\text {bcd }}\end{array}$ & $\begin{array}{l}802.7 \pm \\
16.8^{\text {ab }}\end{array}$ & $\begin{array}{l}1473.5 \pm \\
123.8^{\mathrm{d}}\end{array}$ & $\begin{array}{l}1092.0 \pm \\
19.7^{\text {cd }}\end{array}$ & $\begin{array}{l}710.8 \pm \\
15.2^{\mathrm{a}}\end{array}$ & $\begin{array}{l}1289.7 \pm \\
414.5^{\mathrm{d}}\end{array}$ & $\begin{array}{l}1118.9 \pm \\
121.9^{\text {bcd }}\end{array}$ & $\begin{array}{l}796.8 \pm \\
33.6^{\mathrm{abc}}\end{array}$ & $\begin{array}{l}901.9 \pm \\
69.0^{\text {abcd }}\end{array}$ & $\begin{array}{l}992.9 \pm \\
101.3^{\mathrm{bcd}}\end{array}$ \\
\hline 10 & $\begin{array}{l}\text { 2-methyl-1-butanol +3- } \\
\text { methyl-1-butanol }\end{array}$ & $\begin{array}{l}18238.1 \pm \\
3147.1^{\text {ab }}\end{array}$ & $\begin{array}{l}18197.0 \pm \\
2776.9^{\text {ab }}\end{array}$ & $\begin{array}{l}17793.9 \pm \\
1257.0^{\mathrm{ab}}\end{array}$ & $\begin{array}{l}14573.2 \pm \\
124.8^{\mathrm{a}}\end{array}$ & $\begin{array}{l}22631.7 \pm \\
626.2^{\mathrm{b}}\end{array}$ & $\begin{array}{l}18113.6 \pm \\
109.2^{\mathrm{ab}}\end{array}$ & $\begin{array}{l}14755.3 \pm \\
319.4^{\mathrm{a}}\end{array}$ & $\begin{array}{l}23086.8 \pm \\
6907.8^{\mathrm{b}}\end{array}$ & $\begin{array}{l}20141.1 \pm \\
480.0^{\mathrm{b}}\end{array}$ & $\begin{array}{l}16260.9 \pm \\
461.6^{\text {ab }}\end{array}$ & $\begin{array}{l}17323.4 \pm \\
1686.8^{\text {ab }}\end{array}$ & $\begin{array}{l}17852.8 \pm \\
1828.8^{\text {ab }}\end{array}$ \\
\hline 11 & furfuryl alcohol & $\begin{array}{l}44.2 \pm \\
12.2^{\mathrm{abcd}}\end{array}$ & $\begin{array}{l}48.3 \pm \\
10.3^{\mathrm{bcd}}\end{array}$ & $45.4 \pm 0.5^{\mathrm{bcd}}$ & $\begin{array}{l}37.4 \pm \\
2.6^{\mathrm{abc}}\end{array}$ & $56.1 \pm 1.9^{\mathrm{cd}}$ & $44.3 \pm 1.9^{\mathrm{bcd}}$ & $29.9 \pm 0.2^{\mathrm{a}}$ & $\begin{array}{l}46.9 \pm \\
13.5^{\text {abcd }}\end{array}$ & $\begin{array}{l}45.0 \pm \\
4.8^{\text {bcd }}\end{array}$ & $32.9 \pm 1.5^{\mathrm{ab}}$ & $39.0 \pm 3.0^{\mathrm{abc}}$ & $97.0 \pm 7.6^{\mathrm{d}}$ \\
\hline \multirow[t]{2}{*}{12} & 2-phenylethanol & $\begin{array}{l}23056.5 \pm \\
1501.2^{\mathrm{bc}}\end{array}$ & $\begin{array}{l}20874.6 \pm \\
341.4^{\text {abc }}\end{array}$ & $\begin{array}{l}20867.5 \pm \\
2103.7^{\mathrm{abc}}\end{array}$ & $\begin{array}{l}17231.3 \pm \\
353.0^{\mathrm{a}}\end{array}$ & $\begin{array}{l}27280.9 \pm \\
3401.7^{c}\end{array}$ & $\begin{array}{l}21707.8 \pm \\
676.7^{\text {abc }}\end{array}$ & $\begin{array}{l}18805.0 \pm \\
380.2^{\mathrm{ab}}\end{array}$ & $\begin{array}{l}24556.2 \pm \\
6547.2^{\mathrm{abc}}\end{array}$ & $\begin{array}{l}23564.3 \pm \\
150.5^{\mathrm{bc}}\end{array}$ & $\begin{array}{l}19709.9 \pm \\
790.3^{\text {abc }}\end{array}$ & $\begin{array}{l}20445.4 \pm \\
2005.2^{\text {abc }}\end{array}$ & $\begin{array}{l}19428.1 \pm \\
1824.2^{\mathrm{ab}}\end{array}$ \\
\hline & Monoterpenic alcohols & & & & & & & & & & & & \\
\hline \multirow[t]{2}{*}{13} & Linalool & $89.7 \pm 5.6^{c}$ & $70.8 \pm 2.0^{\mathrm{abc}}$ & $69.8 \pm 6.0^{\mathrm{abc}}$ & $60.7 \pm 1.2^{\mathrm{a}}$ & $95.3 \pm 14.3^{\mathrm{c}}$ & $77.0 \pm 3.5^{\mathrm{bc}}$ & $\begin{array}{l}68.7 \pm \\
0.5^{\text {abc }} \\
\end{array}$ & $\begin{array}{l}77.9 \pm \\
18.6^{\text {abc }} \\
\end{array}$ & $86.7 \pm 3.7^{c}$ & $71.4 \pm 0.2^{\mathrm{abc}}$ & $69.8 \pm 3.5^{\mathrm{abc}}$ & $63.2 \pm 3.8^{\mathrm{ab}}$ \\
\hline & Acids & & & & & & & & & & & & \\
\hline 14 & acetic acid & $\begin{array}{l}202.7 \pm \\
54.1^{\mathrm{cd}}\end{array}$ & $\begin{array}{l}199.9 \pm \\
93.1^{\text {bcd }}\end{array}$ & $\begin{array}{l}154.3 \pm \\
7.9^{\text {bcd }}\end{array}$ & $\begin{array}{l}111.7 \pm \\
8.7^{\mathrm{abc}}\end{array}$ & $\begin{array}{l}225.8 \pm \\
64.1^{\mathrm{d}}\end{array}$ & $\begin{array}{l}179.3 \pm \\
25.7^{\mathrm{cd}}\end{array}$ & $85.7 \pm 0.1^{\mathrm{a}}$ & $189.5 \pm 7.1^{\mathrm{d}}$ & $\begin{array}{l}99.2 \pm \\
17.6^{\mathrm{ab}}\end{array}$ & $81.3 \pm 22.2^{\mathrm{a}}$ & $\begin{array}{l}118.0 \pm \\
21.1^{\mathrm{abc}}\end{array}$ & $\begin{array}{l}136.9 \pm \\
13.4^{\text {abcd }}\end{array}$ \\
\hline 15 & isovaleric acid & $\begin{array}{l}95.5 \pm \\
25.9^{\mathrm{ab}}\end{array}$ & $\begin{array}{l}94.6 \pm \\
19.6^{\mathrm{ab}}\end{array}$ & $93.2 \pm 3.4^{\mathrm{ab}}$ & $72.5 \pm 2.4^{\mathrm{a}}$ & $\begin{array}{l}123.2 \pm \\
11.7^{\mathrm{b}}\end{array}$ & $99.0 \pm 1.4^{\mathrm{ab}}$ & $76.7 \pm 1.3^{\mathrm{ab}}$ & $\begin{array}{l}124.8 \pm \\
35.4^{\mathrm{ab}}\end{array}$ & $\begin{array}{l}89.4 \pm \\
12.1^{\text {ab }}\end{array}$ & $75.9 \pm 1.3^{\mathrm{a}}$ & $100.0 \pm 5.2^{\mathrm{ab}}$ & $97.0 \pm 7.6^{\mathrm{ab}}$ \\
\hline 16 & hexanoic acid & $\begin{array}{l}349.4 \pm \\
40.0^{\text {ab }}\end{array}$ & $\begin{array}{l}387.8 \pm \\
26.4^{\mathrm{ab}}\end{array}$ & $359.7 \pm 2.5^{\mathrm{ab}}$ & $\begin{array}{l}305.5 \pm \\
13.6^{\mathrm{a}}\end{array}$ & $\begin{array}{l}462.6 \pm \\
46.1^{\mathrm{b}}\end{array}$ & $\begin{array}{l}369.4 \pm \\
15.5^{\text {ab }}\end{array}$ & $290.9 \pm 9.1^{\mathrm{a}}$ & $352.3 \pm 0.0^{\mathrm{ab}}$ & $\begin{array}{l}364.8 \pm \\
22.0^{\mathrm{ab}}\end{array}$ & $\begin{array}{l}326.9 \pm \\
28.0^{\mathrm{a}}\end{array}$ & $365.2 \pm 0.0^{\mathrm{ab}}$ & $\begin{array}{l}336.3 \pm \\
61.2^{\mathrm{ab}}\end{array}$ \\
\hline 17 & octanoic acid & $\begin{array}{l}2304.3 \pm \\
52.2^{\mathrm{c}}\end{array}$ & $\begin{array}{l}2012.7 \pm \\
94.9^{\text {abc }}\end{array}$ & $\begin{array}{l}1857.2 \pm \\
67.2^{\mathrm{ab}}\end{array}$ & $\begin{array}{l}1505.8 \pm \\
93.5^{\mathrm{a}}\end{array}$ & $\begin{array}{l}2699.1 \pm \\
435.3^{\mathrm{c}}\end{array}$ & $\begin{array}{l}2192.1 \pm \\
117.3^{\text {abc }}\end{array}$ & $\begin{array}{l}2027.0 \pm \\
49.9^{\text {abc }}\end{array}$ & $\begin{array}{l}2187.8 \pm \\
467.4^{\mathrm{abc}}\end{array}$ & $\begin{array}{l}2336.5 \pm \\
105.9^{\mathrm{c}}\end{array}$ & $\begin{array}{l}2178.9 \pm \\
83.3^{\text {abc }}\end{array}$ & $\begin{array}{l}2217.8 \pm \\
98.4^{\mathrm{bc}}\end{array}$ & $\begin{array}{l}2079.6 \pm \\
81.3^{\text {abc }}\end{array}$ \\
\hline \multirow[t]{2}{*}{18} & decanoic acid & $328.8 \pm 8.6^{\mathrm{fg}}$ & $\begin{array}{l}245.5 \pm \\
3.6^{\mathrm{cd}}\end{array}$ & $183.0 \pm 4.6^{\mathrm{ab}}$ & $\begin{array}{l}134.0 \pm \\
11.1^{\mathrm{a}}\end{array}$ & $\begin{array}{l}380.7 \pm \\
72.1^{\mathrm{fg}}\end{array}$ & $\begin{array}{l}259.4 \pm \\
15.2^{\mathrm{de}}\end{array}$ & $\begin{array}{l}211.9 \pm \\
16.8^{\mathrm{bc}}\end{array}$ & $\begin{array}{l}194.1 \pm \\
30.8^{\mathrm{ab}}\end{array}$ & $\begin{array}{l}354.8 \pm \\
13.2^{g}\end{array}$ & $292.6 \pm 8.5^{\mathrm{ef}}$ & $\begin{array}{l}257.4 \pm \\
13.5^{\mathrm{de}}\end{array}$ & $213.1 \pm 4.9^{\mathrm{b}}$ \\
\hline & Furan compounds & & & & & & & & & & & & \\
\hline \multirow[t]{2}{*}{19} & Furfural & - & $74.9 \pm 2.7^{\mathrm{a}}$ & $\begin{array}{l}153.1 \pm \\
27.0^{\text {cd }}\end{array}$ & $\begin{array}{l}188.9 \pm \\
6.3^{\mathrm{ef}}\end{array}$ & - & $\begin{array}{l}100.6 \pm \\
3.2^{\mathrm{ab}}\end{array}$ & $\begin{array}{l}183.1 \pm \\
1.1^{\mathrm{de}}\end{array}$ & $397.6 \pm 96.2^{g}$ & - & $\begin{array}{l}113.2 \pm \\
5.3^{\mathrm{bc}}\end{array}$ & $224.5 \pm 32.9^{f}$ & $\begin{array}{l}360.7 \pm \\
31.8^{g}\end{array}$ \\
\hline & Lactones & & & & & & & & & & & & \\
\hline 20 & cis-oak lactone & - & $102.3 \pm 2.7^{\mathrm{a}}$ & $205.1 \pm 6.2^{\mathrm{cd}}$ & $\begin{array}{l}274.3 \pm \\
12.7^{\mathrm{e}}\end{array}$ & - & $\begin{array}{l}131.4 \pm \\
4.3^{\mathrm{bc}}\end{array}$ & $\begin{array}{l}260.7 \pm \\
1.5^{\mathrm{de}}\end{array}$ & $429.8 \pm 90.9^{f}$ & - & $\begin{array}{l}124.6 \pm \\
2.4^{\text {ab }}\end{array}$ & $271.8 \pm 9.6^{\mathrm{e}}$ & $\begin{array}{l}377.9^{ \pm} \\
15.4^{\mathrm{f}}\end{array}$ \\
\hline
\end{tabular}




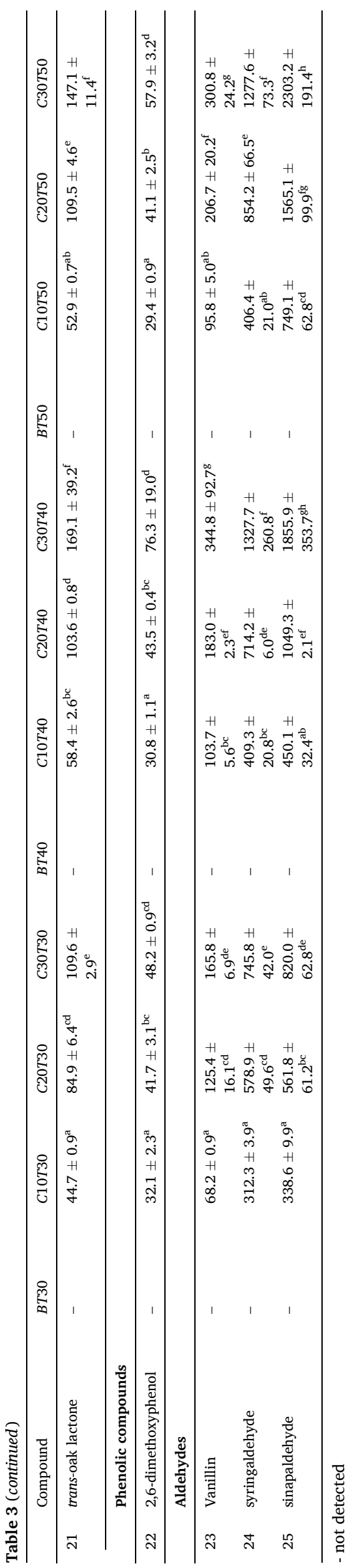

yield was observed for furfural, namely $27(\mu \mathrm{g} / \mathrm{L}) / \mathrm{g}$, and similar extraction yield was observed for 5-methylfurfural, accounting for 11.2 $(\mu \mathrm{g} / \mathrm{L}) / \mathrm{g}$. Focusing oak-lactones, Bautista-Ortín and collaborators reported maximum concentration of $300 \mu \mathrm{g} / \mathrm{L}$ of cis-oak lactone and 130 $\mu \mathrm{g} / \mathrm{L}$ of trans-oak lactone, accounting for extractions yields of 115 $(\mu \mathrm{g} / \mathrm{L}) / \mathrm{g}$ and $50(\mu \mathrm{g} / \mathrm{L}) / \mathrm{g}$, respectively. In the present work, concentrations of cis- and trans-oak lactone were $360 \mu \mathrm{g} / \mathrm{L}$ and $144 \mu \mathrm{g} / \mathrm{L}$, accounting for lower extraction yields of $30(\mu \mathrm{g} / \mathrm{L}) / \mathrm{g}$ and $12(\mu \mathrm{g} / \mathrm{L}) / \mathrm{g}$, respectively, attained with previously used wood. Bautista-Ortín and collaborators also reported maximum vanillin concentration of $443 \mu \mathrm{g} / \mathrm{L}$ corresponding to a yield of $170(\mu \mathrm{g} / \mathrm{L}) / \mathrm{g}$, whereas in the present work a similar concentration of vanillin of $480 \mu \mathrm{g} / \mathrm{L}$ was attained with used wood, but corresponding to a lower yield of $40(\mu \mathrm{g} / \mathrm{L}) / \mathrm{g}$. Lastly, Bautista-Ortín and collaborators reported concentrations of 4-ethylphenol and 4-ethylguaiacol of $900 \mu \mathrm{g} / \mathrm{L}$ and $60 \mu \mathrm{g} / \mathrm{L}$ respectively, which are often considered spoilage in wine, whereas in the presented work such compounds were absent. More recently, Dumitriu and collaborators also applied American wood chips for wine ageing, in stainless steel tanks, at $3 \mathrm{~g} / \mathrm{L}$ and $5 \mathrm{~g} / \mathrm{L}$, for a maximum of 3 months. For the $3 \mathrm{~g} / \mathrm{L}$ wood chips concentration, The authors reported higher concentrations of furfural $(900 \mu \mathrm{g} / \mathrm{L})$ and 5-methylfurfural (about $400 \mu \mathrm{g} / \mathrm{L}$ ), and similar concentrations of cis-oak lactone $(400 \mu \mathrm{g} / \mathrm{L})$ and trans-oak lactone $(130$ $\mu \mathrm{g} / \mathrm{L}$ ) (Dumitriu et al., 2019). Lastly, focusing on grape marc spirits, Rodríguez-Solana and collaborators reported significantly higher concentrations of vanillin and oak lactone in different grape marks aged in American oak barrels (Rodriguez-Solana et al., 2017), using an optimized process.

On a global analysis, beer was the beverage in which the additive effect of used wood was more pronounced, regarding extraction of characteristic wood volatiles as well as transference of wine compounds previously adsorbed by wood. Moreover, beer presented the most satisfactory extraction yields when compared with those reported in the literature. Therefore, it was considered as the more advantageous matrix for the application of reused wood and chosen for the development of formulation and preference studies.

\subsection{Impact of used oak wood on the sensory profile of beer}

Several formulations using different binomial combinations of wood concentration and temperature were prepared and presented to a panel of trained tasters, resorting to the same wood previously put in contact with fortified wine. Samples were analyzed by GC-MS, in order to search for correlations between volatile composition and aroma preference. Characterization of volatile composition of the different beer samples is presented in Table 3 and the sensory evaluation of the correspondent samples is presented in Fig. 1. Again, the main adsorbed wine volatiles extracted to beer were ethyl lactate, diethyl succinate and diethyl malate, which were originally absent from beer, along with characteristic wood extractives, vanillin, cis- and trans-oak lactone, syringaldehyde, sinapaldehyde, furfural and 2,6-dimethoxyphenol. Concentration of wood affected significantly the incorporation of volatile compounds in beer, either regarding adsorbed wine volatiles or characteristic wood extractives, due to the increasing extractable amount supplied by higher wood to beer ratios. Mathematical models established in previous work have demonstrated that temperature mainly increases extraction of characteristic wood compounds, having low to null impact on the extraction of adsorbed wine volatiles (Coelho, Teixeira, et al., 2019). In fact, very low variation was observed for ethyl lactate, diethyl succinate and diethyl malate comparing the different temperatures for the same wood concentration. On the other hand, noticeable variations were observed for the extraction of characteristic wood extractives, when comparing application of similar wood concentrations at different temperatures. Statistically significant differences were found when applying the same wood concentration at different temperatures for furfural, oak lactones, vanillin, syringaldehyde and sinapaldehyde, with higher compound concentrations at upper temperature. Thus, results 
a)

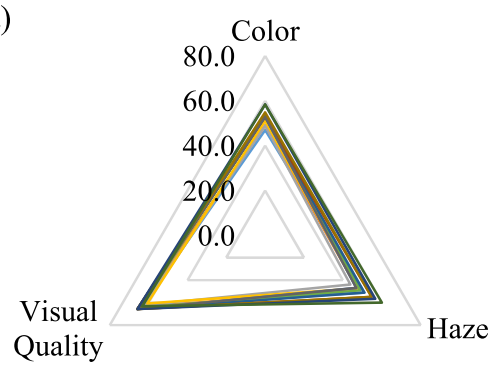

c)

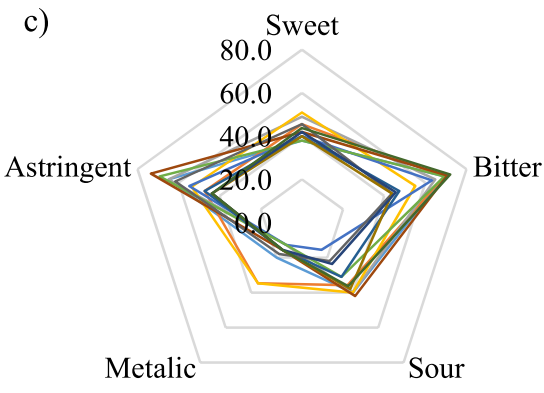

b)

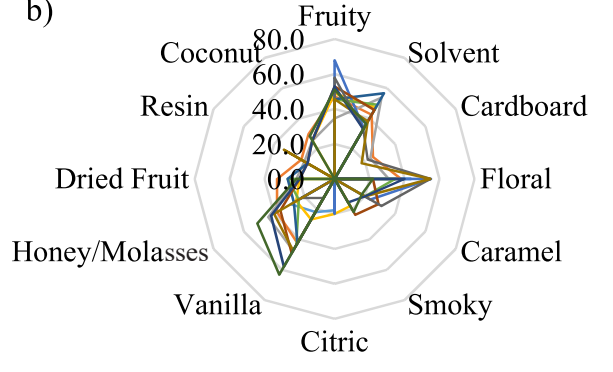

d)

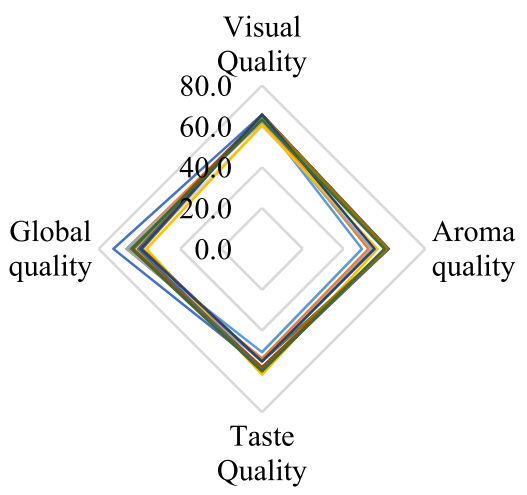

Fig. 1. Sensory evaluation of the different beer formulations regarding a) visual descriptors, b) aroma descriptors, c) taste descriptors and d) overall quality. Samples are represented by different colors $(-$ BT30 - BT40 - BT50 $=$ C10T30

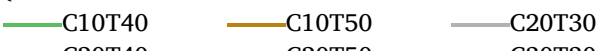
-C20T40 C20T50 C30T30 -C30T40 _C30T50) identified by a CXTy key, where $C x$ refers to wood concentration at $x \mathrm{~g} / \mathrm{L}$ and $T y$ refers to contact temperature performed at $y{ }^{\circ} \mathrm{C}$. Controls of beer incubated at the same temperatures without addition of wood were also performed, identified as BTy, where $y$ refers to contact temperature in ${ }^{\circ} \mathrm{C}$. obtained are also in good agreement with the established in a previous work (Coelho, Teixeira, et al., 2019). Wood sorption was again observed, with decreases in beer volatile compound concentrations by application of oak wood. Esters, either acetates (isoamyl and 2-phenylethyl) or ethyl esters (hexanoate, octanoate and decanoate), acids (octanoic and decanoic) and linalool were found to adsorb in wood. Statistical significant differences were more prominent when comparing beer controls without wood with beer in contact with the highest concentration of wood (30 g/L). Ramirez-Ramirez and collaborators also reported wood sorption of linalool, ethyl octanoate and isoamyl acetate from synthetic wine matrices, again with increased sorption for higher wood concentrations, being the obtained results in good agreement with the previously reported (Ramirez-Ramirez et al., 2001). Regarding the effect of temperature, statistical significant differences were only observed for ethyl octanoate and decanoate concentrations at $50{ }^{\circ} \mathrm{C}$ when compared with the remaining temperatures, which was observed for beers with and without wood. Thus, temperature possibly affected compounds stability, rather than sorption by wood. On the other hand, decrease in octanoic and decanoic acid concentrations was more pronounced for beer in contact with wood at $30^{\circ} \mathrm{C}$, suggesting an impact of temperature on their sorption.

Focusing on the sensory analyses of beer formulations, several different features can be highlighted. Descriptors were grouped in visual, aroma and taste attributes, according to the evaluated sensory properties. For a better interpretation of the results, a multivariate PCA analysis was performed in order to distinguish sensory features of the tasted samples, presented in Fig. 2a, and in a more detailed analysis, correlation between aroma descriptors and volatile composition of samples is presented in Fig. 2b. Regarding visual descriptors, a yellow color was described for beers put in contact with wood at $30{ }^{\circ} \mathrm{C}$ and golden for samples put in contact with wood at $40{ }^{\circ} \mathrm{C}$ and $50{ }^{\circ} \mathrm{C}$. Haze was noticeable in all the evaluated samples, which was slightly higher in beers put in contact with wood at higher temperatures of $40{ }^{\circ} \mathrm{C}$ and 50 ${ }^{\circ} \mathrm{C}$. Despite small variations, overall visual quality was similar for all the tasted samples.

Focusing aroma characteristics, fruity, solvent and floral were the descriptors with the higher overall ratings by the panelists for beer samples, as well as vanilla for the beers put in contact with wood. Higher fruity scores were attributed to beers without application of wood, especially for $40{ }^{\circ} \mathrm{C}$ and $50{ }^{\circ} \mathrm{C}$, as visible in the scatterplot presented in Fig. 2a. The lower fruity scores of beers put in contact with wood can be correlated with adsorption of several esters, such as isoamyl acetate (perception threshold, $P T$, of $30 \mu \mathrm{g} / \mathrm{L}$ (Christoph \& Bauer-Christoph, 2007)), ethyl octanoate (PT of $260 \mu \mathrm{g} / \mathrm{L}$ (Christoph \& Bauer-Christoph, 2007)) and ethyl decanoate (PT of $50 \mu \mathrm{g} / \mathrm{L}$ (Christoph \& Bauer-Christoph, 2007)) as previously discussed. In fact, Fig. 2b demonstrates correlation of these esters with perception of fruity aroma by tasters, which were more prominent in control beers and less in beers put in contact with wood. Additional esters were extracted from wood, namely ethyl lactate (PT of $100 \mathrm{mg} / \mathrm{L}$ (Christoph \& Bauer-Christoph, 2007)), diethyl succinate (PT of $100 \mathrm{mg} / \mathrm{L}$ (Christoph \& Bauer-Christoph, 2007)) and diethyl malate, but their concentrations were low considering the perception threshold for each compound.

Similarly to fruity aromas, scores of floral descriptors were also lower in beers put in contact with wood. Several compounds associated with floral descriptors, namely 2-phenylethyl acetate (PT of $250 \mu \mathrm{g} / \mathrm{L}$ (Christoph \& Bauer-Christoph, 2007)), 2-phenylethanol (PT of $10 \mathrm{mg} / \mathrm{L}$ (Christoph \& Bauer-Christoph, 2007)) and linalool (PT of $0.14 \mu \mathrm{g} / \mathrm{L}$ (Christoph \& Bauer-Christoph, 2007)), were found in beer samples. In fact, linalool and 2-phenylethyl acetate concentrations decreased significantly with application of wood, again due to wood sorption, leading to a lower perception of floral aroma. Several descriptors commonly associated with aged wood products were also found. Vanilla, coconut and smoky descriptors were correlated with beers put in contact with wood, and more prominent in the contacts performed at higher temperatures of $40^{\circ} \mathrm{C}$ and $50^{\circ} \mathrm{C}$. This is coherent with the higher extraction of characteristic wood volatiles such as vanillin, cis- and trans-oak lactone, 2,6-dimethoxyphenol, syringaldehyde, sinapaldehyde and furfural, often associated with barrel ageing descriptors. The contribution of temperature is furthermore highlighted by the perception of different binomial formulations by the panelists. Formulations using lower wood concentrations and higher temperatures had similar perception of wood descriptors of formulations using higher wood amounts and lower contact temperatures. Considering that extraction of adsorbed wine volatiles migrating to beer through wood is not affected by temperature, the use of different wood concentration/temperature 
a)
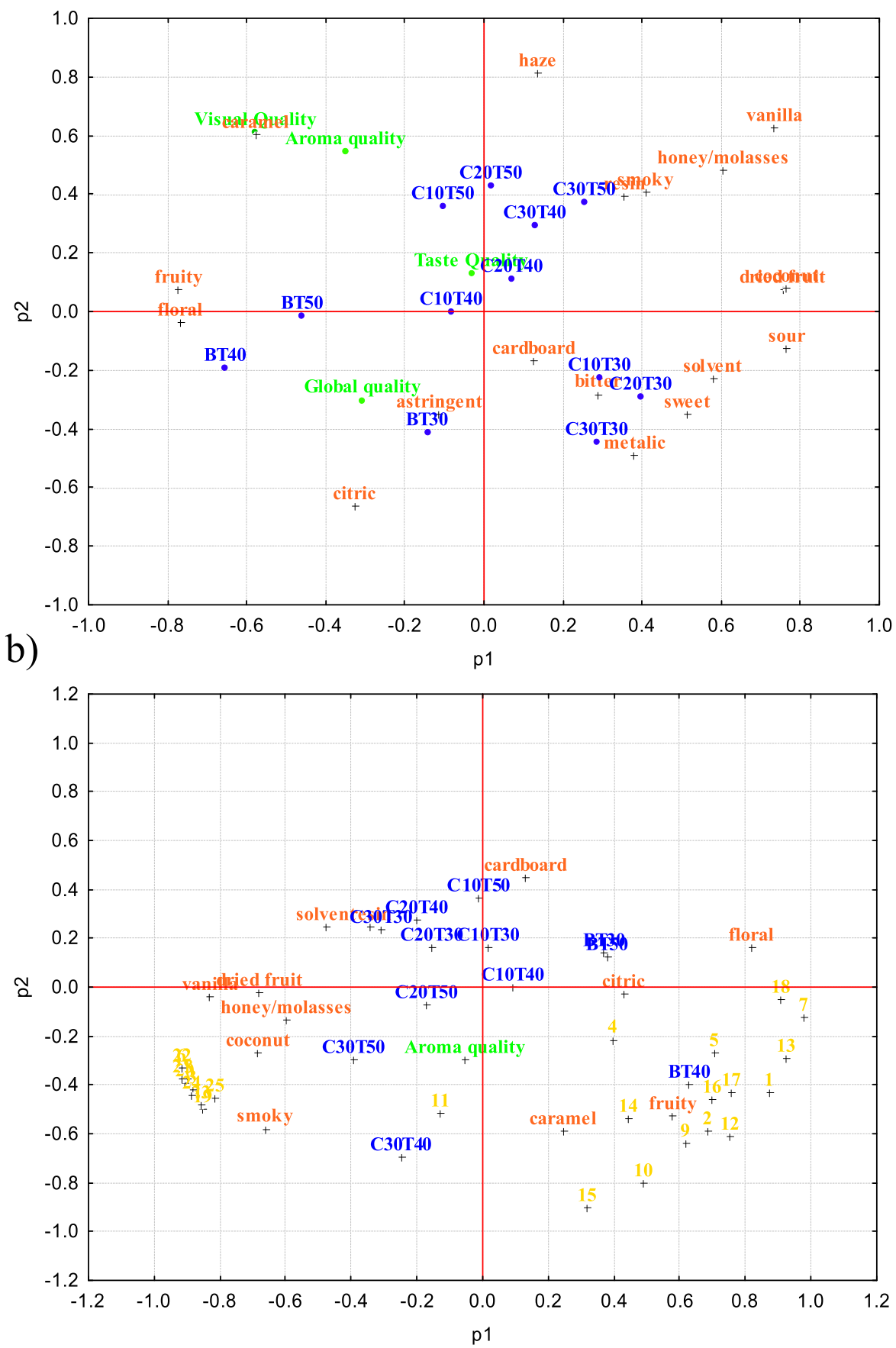

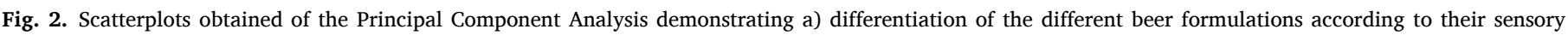

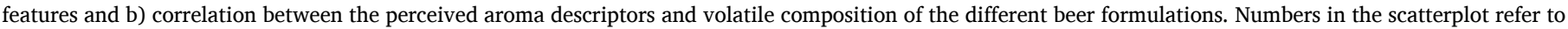
the volatile compounds presented in Table 3.

binomials allows further fine tuning of the intended aromatic bouquet, as previously hypothesized (Coelho, Teixeira, et al., 2019). Despite the presence of volatile fatty acids, no faulty aromas were perceived by the trained panelists. Focusing on taste quality, beers were marked mainly by perception of bitterness and astringency. Astringency was overall higher in control beers and beers placed with wood at low to moderate temperatures of $30^{\circ} \mathrm{C}$ and $40{ }^{\circ} \mathrm{C}$, especially for sample C30T40. This was also observed for bitterness, with the exception of sample C30T50. Sweetness was higher for samples put at $30{ }^{\circ} \mathrm{C}$ but overall variation of sweetness perception was rather low. Also sour descriptors were more associated with beers placed at $30{ }^{\circ} \mathrm{C}$ but no significant trends could be observed. Perception of metallic taste was low for the overall beers, being higher only for С10T30 and С30T30 samples. Focusing on quality perception by the panelists, visual quality was similar for all the tasted samples, being only slightly lower for beers placed with wood at $30^{\circ} \mathrm{C}$. Higher aroma quality was attributed to beers with greater perception of wood descriptors, when compared with beer controls without wood. Moderate variations were also observed for taste quality, which was considered a little higher for beers placed with wood at temperatures of $40{ }^{\circ} \mathrm{C}$ and $50^{\circ} \mathrm{C}$. Panelists attributed slightly higher global quality scores 
to control beers, probably considering them a more balanced or recognizable product, with more pronounced fruity and floral aromas, taking into account what would be expected from the corresponding beer type.

\section{Conclusions}

Due to the reuse, wood transfers compounds from one beverage to another, along with a significant amount of wood extractives. The impact of wood reuse is strongly dependent on the beverages to be aged and their chemical composition. Transference of compounds from the previous beverage is perceived in the sensory characteristics of beer aged with reused wood. Wood also adsorbs compounds from the beverages during contact, which further modifies the perceived sensory features. Different binomial combinations of wood concentration and contact temperature lead to differentiated volatile compositions and consequently different organoleptic characteristics. Higher wood concentrations and contact temperatures lead to a sensory profile more marked by vanilla and coconut descriptors, whereas lower wood concentrations lead to more dominant fruity and floral characteristics. Reuse of oak chips is a feasible alternative for additive and subtractive ageing of alcoholic beverages, to be incorporated in accelerated ageing processes.

\section{CRediT authorship contribution statement}

Eduardo Coelho: Conceptualization, Methodology, Formal analysis, Investigation, Writing - original draft, Writing - review \& editing, Visualization. José A. Teixeira: Resources, Writing - review \& editing, Project administration, Funding acquisition. Teresa Tavares: Writing review \& editing, Supervision, Project administration, Funding acquisition. Lucília Domingues: Resources, Writing - review \& editing, Supervision, Project administration, Funding acquisition. José M. Oliveira: Conceptualization, Resources, Writing - review \& editing, Supervision, Project administration.

\section{Declaration of competing interest}

The authors declare that they have no known competing financial interests or personal relationships that could have appeared to influence the work reported in this paper.

\section{Acknowledgements}

This study was supported by the Portuguese Foundation for Science and Technology (FCT) under the scope of the strategic funding of UIDB/ 04469/2020 unit and BioTecNorte operation (NORTE-01-0145-FEDER000004) funded by the European Regional Development Fund under the scope of Norte2020 - Programa Operacional Regional do Norte. Fermentum - Engenharia das Fermentações Lda. also participated in cofunding and provided beer samples used in this work, which authors would like to acknowledge. Lastly, authors would like to thank Mr. Benoît Verdier and Seguin Moreau for supplying the woods and Mr. Paulo Coutinho and Quinta do Portal for supplying the fortified wine.

\section{References}

Álamo-Sanza, M., \& Nevares, I. (2018), Oak wine barrel as an active vessel: A critical review of past and current knowledge. Critical Reviews in Food Science and Nutrition, $58,2711-2726$.

Barrera-García, V. D., Gougeon, R. D., Di Majo, D., De Aguirre, C., Voilley, A., \& Chassagne, D. (2007). Different sorption behaviors for wine polyphenols in contact with oak wood. Journal of Agricultural and Food Chemistry, 55, 7021-7027.

Bautista-Ortín, A. B., Lencina, A. G., Cano-López, M., Pardo-Mínguez, F., LópezRoca, J. M., \& Gómez-Plaza, E. (2008). The use of oak chips during the ageing of a red wine in stainless steel tanks or used barrels: Effect of the contact time and size of the oak chips on aroma compounds. Australian Journal of Grape and Wine Research, $14,63-70$.
Canas, S., Caldeira, I., Anjos, O., \& Belchior, A. P. (2019). Phenolic profile and colour acquired by the wine spirit in the beginning of ageing: Alternative technology using micro-oxygenation vs traditional technology. LWT - Food Science and Technology, $111,260-269$.

Canas, S., Caldeira, I., \& Belchior, A. P. (2013). Extraction/oxidation kinetics of low molecular weight compounds in wine brandy resulting from different ageing technologies. Food Chemistry, 138, 2460-2467.

Chatonnet, P. (2007). Produits alternatifs à 1 'élevage en barriques lére partie : Utilisation dans le monde et tipologies. Revue Internet de Viticulture et Oenologie, (19), $1-5$

Christoph, N., \& Bauer-Christoph, C. (2007). Flavour of spirit drinks: Raw materials, fermentation, distillation, and ageing. In Flavours and fragrances: Chemistry, bioprocessing and sustainability (pp. 219-239).

Coelho, E., Azevedo, M., Teixeira, J. A., Tavares, T., Oliveira, J. M., \& Domingues, L. (2020). Evaluation of multi-starter S. cerevisiae/D. bruxellensis cultures for mimicking and accelerating transformations occurring during barrel ageing of beer. Food Chemistry, 323, 126826.

Coelho, E., Domingues, L., Teixeira, J. A., Oliveira, J. M., \& Tavares, T. (2019). Understanding wine sorption by oak wood: Modeling of wine uptake and characterization of volatile compounds retention. Food Research International, 116, 249-257.

Coelho, E., Teixeira, J. A., Domingues, L., Tavares, T., \& Oliveira, J. M. (2019). Factors affecting extraction of adsorbed wine volatile compounds and wood extractives from used oak wood. Food Chemistry, 295, 156-164.

Coetzee, C., \& Du Toit, W. J. (2015). Sauvignon blanc wine: Contribution of ageing and oxygen on aromatic and non-aromatic compounds and sensory composition: A review. South African Journal of Enology and Viticulture, 36, 347-365.

Doussot, F., De Jéso, B., Quideau, S., \& Pardon, P. (2002). Extractives content in cooperage oak wood during natural seasoning and toasting; influence of tree species, geographic location, and single-tree effects. Journal of Agricultural and Food Chemistry, 50, 5955-5961.

Dumitriu, G. D., Teodosiu, C., Gabur, I., Cotea, V. V., Peinado, R. A., \& de Lerma, N. L. (2019). Evaluation of aroma compounds in the process of wine ageing with oak chips. Foods, 8, 1-19.

García-Alcaraz, J. L., Flor Montalvo, F. J., Martínez Cámara, E., Pérez de la Parte, M. M., Jiménez-Macías, E., \& Blanco-Fernández, J. (2020). Economic-environmental impact analysis of alternative systems for red wine ageing in re-used barrels. Journal of Cleaner Production, 244, Article 118783.

García-Estévez, I., Alcalde-Eon, C., Le Grottaglie, L., Rivas-Gonzalo, J. C., \& EscribanoBailón, M. T. (2015). Understanding the ellagitannin extraction process from oak wood. Tetrahedron, 71, 3089-3094.

International Organization for Standardization. (1994). Sensory analysis. Identification and selection of descriptors for establishing a sensory profile by a multidimensional approach (ISO 11035:1994).

Mosedale, J. R., \& Puech, J.-L. (2003). BARRELS | wines, spirits, and other beverages. In Encyclopedia of food sciences and nutrition (pp. 393-403).

National Center for Biotechnology Information. (2020). PubChem compound database. Retrieved April 17, 2020, from https://pubchem.ncbi.nlm.nih.gov/.

Pielech-Przybylska, K., \& Balcerek, M. (2019). New trends in spirit beverages production. In Alcoholic beverages.

Quinn, D. (2014). Irish whiskey. Whisky, 7-16.

Ramirez-Ramirez, G., Lubbers, S., Charpentier, C., Feuillat, M., Voilley, A., \& Chassagne, D. (2001). Aroma compound sorption by oak wood in a model wine. Journal of Agricultural and Food Chemistry, 49, 3893-3897.

Rodriguez-Solana, R., Rodriguez-Freigedo, S., Salgado, J. M., Domínguez, J. M., \& Cortés-Diéguez, S. (2017). Optimisation of accelerated ageing of grape marc distillate on amicro-scale process using a box-benhken design: Influence of oak origin, fragment size and toast level on the composition of the final product. Australian Journal of Grape and Wine Research, 23, 5-14.

Roullier-Gall, C., Signoret, J., Hemmler, D., Witting, M. A., Kanawati, B., Schäfer, B., \& Schmitt-Kopplin, P. (2018). Usage of FT-ICR-MS metabolomics for characterizing the chemical signatures of barrel-aged whisky. Frontiers in Chemistry, 6, 1-11.

Schwarz, M., Rodríguez, M. C., Sánchez, M., Guillén, D. A., \& Barroso, C. G. (2014). Development of an accelerated aging method for Brandy. LWT - Food Science and Technology, 59, 108-114.

Snauwaert, I., Roels, S. P., Van Nieuwerburg, F., Van Landschoot, A., De Vuyst, L., \& Vandamme, P. (2016). Microbial diversity and metabolite composition of Belgian red-brown acidic ales. International Journal of Food Microbiology, 221, 1-11.

Spitaels, F., Wieme, A. D., Janssens, M., Aerts, M., Daniel, H. M., Van Landschoot, A., \& Vandamme, P. (2014). The microbial diversity of traditional spontaneously fermented lambic beer. PloS One, 9.

Sterckx, F. L., Saison, D., \& Delvaux, F. R. (2012a). Wood aging of beer. Part I: Influence on beer flavor and monophenol concentrations. Journal of the American Society of Brewing Chemists, 70, 55-61.

Sterckx, F. L., Saison, D., \& Delvaux, F. R. (2012b). Wood aging of beer. Part II: Influence of wood aging parameters on monophenol concentrations. Journal of the American Society of Brewing Chemists, 70, 62-69.

Tao, Y., García, J. F., \& Sun, D.-W. (2014). Advances in wine aging technologies for enhancing wine quality and accelerating wine aging process. Critical Reviews in Food Science and Nutrition, 54, 817-835.

Wyler, P., Angeloni, L. H. P., Alcarde, A. R., \& da Cruz, S. H. (2015). Effect of oak wood on the quality of beer. Journal of the Institute of Brewing, 121, 62-69.

Zamora, F. (2019). Barrel aging; types of wood. Red Wine Technology, 125-147. 